Mariagiovanna Baccara

Leeat Yariv

\title{
Similarity and Polarization in Groups
}

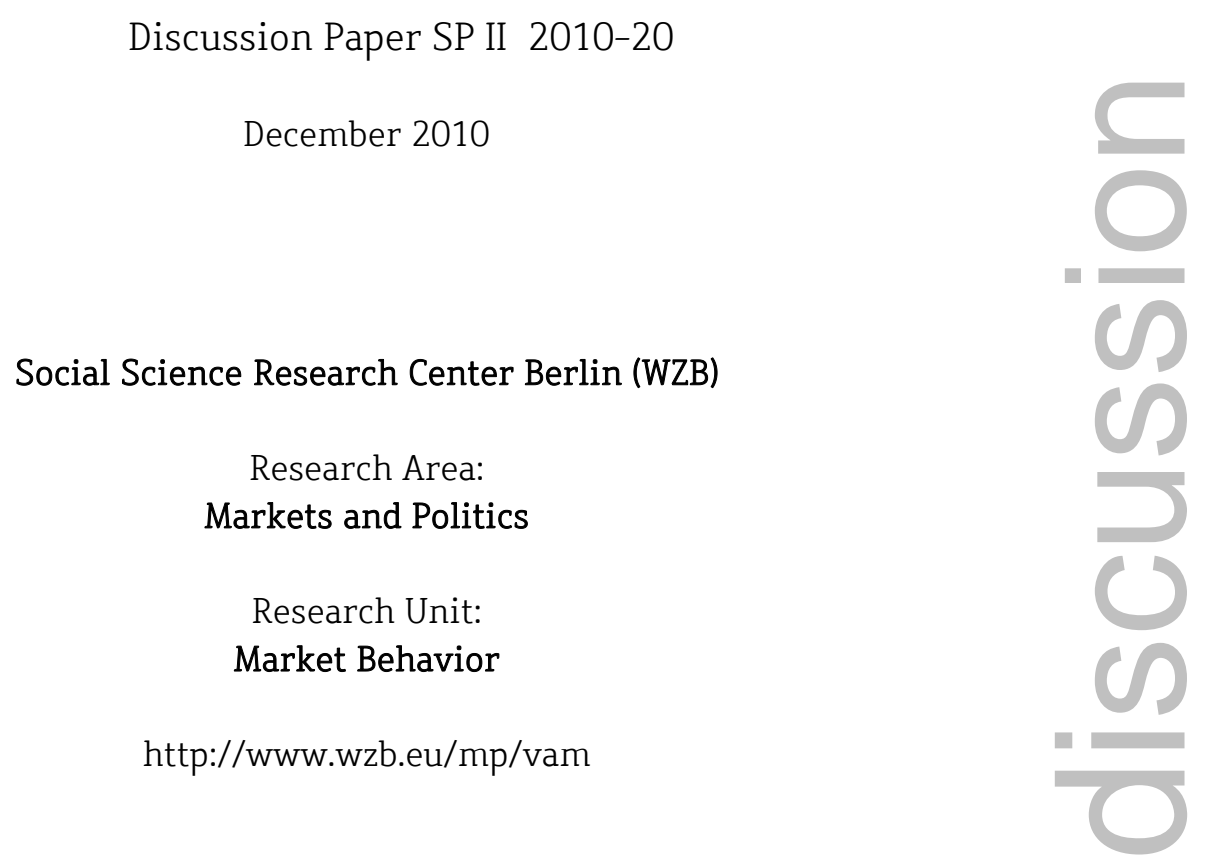

E-mail: mbaccara@wustl.edu 
(c) The copyright remains with the authors

Mariagiovanna Baccara* and Leeat Yariv†

Similarity and Polarization in Groups

Discussion Paper SP II 2010-20

Wissenschaftszentrum Berlin für Sozialforschung (2010)

*Olin School of Business, Washington University. Email: mbaccara@wustl.edu.

†Division of Humanities and Social Sciences, California Institute of Technology. Email: lyariv@hss.caltech.edu. $\neq$ We thank two anonymous referees, the editor Andrea Prat, Heski Bar-Isaac, Jean-Pierre Benoit, Tim Feddersen, Hugo Hopenhayn, Matt Jackson, Alessandro Lizzeri, Andrea Mattozzi, Maggie McConnell, Wolfgang Pesendorfer, Debraj Ray, Ronny Razin, and Bill Zame. Financial support from the National Science Foundation (SES 0963583) is gratefully acknowledged.

Social Science Research Center Berlin (WZB)

Research Area:

Markets and Politics

Research Unit:

Market Behavior
Wissenschaftszentrum Berlin für Sozialforschung gGmbH (WZB)

Forschungsschwerpunkt:

Märkte und Politik

Abteilung:

Verhalten auf Märkten

Reichpietschufer 50, D-10785 Berlin

Telefon: +49 30 25491-0, Fax: +49 30 25491-440

http://www.wzb.eu 


\section{Abstract}

The focus of this paper is the endogenous formation of peer groups. We study a model in which agents choose their peers prior to making decisions on multiple issues. Agents differ in how much they value the decision outcomes on one issue relative to another. While each individual can collect information on at most one issue, all information is shared within the group. Thus, the group's preference composition affects the type of information that gets collected. We characterize stable groups, groups that are optimal for all their members. When information costs are low, stable groups must be sufficiently homogeneous. Furthermore, stability requires more similarity among extremists than among moderate individuals. When information costs are substantial, a free rider problem arises, and makes extreme peers more desirable, as they are more willing to invest in information acquisition. We show that, as information costs grow, polarization appears and becomes increasingly pronounced in stable groups.

JEL classification: D82, D85.

Keywords: Homophily, Polarization, Group Formation, Information Collection 


\section{INTRODUCTION}

There are many realms in which individuals choose whom they interact with, socially and strategically. Individuals choose which Internet forums to participate in, clubs to join, neighborhoods to live in, schools to go to, and so on. In fact, new platforms such as online social networks, blogs, etc. allow users to choose their peers without any physical or geographical constraint. Interestingly, a vast empirical literature in sociology suggests consistent patterns of group formation. Indeed, agents appear to associate with those similar to them (e.g., in demographics, political opinions, or beliefs). ${ }^{1}$ Moreover, in an era in which the Internet penetration rate in North America is $73 \%$ (and $87 \%$ among teenagers), concerns have been raised about the consequences of unconstrained socialization among individuals of similar, and possibly extreme, views. ${ }^{2}$

While, over the decades, the analysis of strategic interactions across domains has received wide attention, theoretically and empirically, the group of players is usually assumed to be determined exogenously. ${ }^{3}$ The focus of the current paper is the analysis of an extended game in which, first, agents choose their group of peers and, second, a strategic interaction (namely, a public good game) takes place. The goal is to understand how the interplay between the group formation stage and the strategic interaction stage determine the properties of the peer groups that arise in equilibrium. In particular, we provide a taxonomy of environments generating groups that consist of similar, heterogenous, or highly polarized agents, in terms of their fundamental characteristics.

We study a model in which agents make individual decisions on two different issues, and their utilities differ in the relative weight they put on each issue. These issues can be a metaphor for lifedecisions regarding parenting and retirement, choices concerning consumption goods for different uses (e.g.; food and books), etc. For example, depending on their personal circumstances and demographic

\footnotetext{
${ }^{1}$ See the literature review for a brief summary of the work on this phenomenon, known as homophily.

${ }^{2}$ The leading online social networks contain a large volume of members. As of mid-2010, MySpace had over 200 million members, while Facebook had over 400 million members. In 2007, Forrester Research estimated that 50\% of teenagers of age 12-17 visited online social networks at least weekly. Since then, analysts have reported persistent growth (see Lipsman (2008)). The public sphere has continuously scrutinized the role of the web in enabling.extreme and intolerant behavior. For example, in 2003 the U.S. Army created the Counterterrorism Center to follow online extremist groups, and in 2006 an Internet Radicalization Task Force was formed by the Homeland Security Policy Institute (see Thomas, 2009). Furthermore, the Columbine shooters reportedly learned how to construct sophisticated bombs through their Internet activity. 'In the massacre's aftermath, some measures have been taken in U.S. jurisdiction to limit minors' access to online communities (e.g., the Children's Internet Protection Act).

${ }^{3}$ See below for a clescription of several exceptions.
} 
characteristics, agents may vary in how much they worry about their children's educational prospects (important for younger individuals) as opposed to their savings (that are very important for individuals nearing retirement). Similarly, depending on their personal tastes and hobbies, they might differ in how much they are concerned with the quality of food they eat relative to the selection of books they read. Each agent's taste is characterized by a parameter in $[0,1]$, proxying for how much she cares about one issue relative to the other.

We assume that there is some uncertainty about the optimal choice on each of the two issues. Prior to making decisions, each individual can exert costly effort in collecting information on, at most, one issue. ${ }^{4}$ Besides gathering information directly, wè assume that individuals have a.ccess to information through their peers. In our model, agents have the possibility of forming groups and what defines a peer group is that the information collected by each member is made public within the group.

For any fixed group of individuals, we characterize the equilibrium choice of information collection, a mapping from the composition of tastes of the agents in the group into the volume of information collected on each issue.

To capture the notion that new technologies allow individuals to connect on platforms that are not bounded by local geography, we then step back and consider the group of peers as an object of choice. Depending on her tastes, and foreseeing the amount and the type of information that is collected within each group, each individual prefers certain peer groups to others. We characterize the peer group's unconstrained optimal composition for each individual's taste. We show that, for each individual, there is a large equivalence class of optimal groups, potentially with maximal variance of tastes.

Stable groups are ones that satisfy natural equilibrium constraints in the group-formation stage. That is, a group is stable if it is optimal for all its members.

Our first main result provides a characterization of stable groups when information gathering has very low costs, or is free. The case of free information is relevant for situations in which some form of information collection is par for the course, and individuals face only a choice on the type of information to gather- e.g., which websites to visit while surfing the Internet (those directed at

\footnotetext{
${ }^{4}$ This is a simplification capturing the idea that agents may face constraints on the effort they can exert on information gathering. As discussed below, the analysis is unchanged if we allow each agent to collect multiple pieces of information.
} 
child-rearding or those focusing on investment advice), which part of the newspaper to read in the morning (the food section or the book reviews), and so on. We show that stable groups of a fixed size are identified by a partition of the taste parameter range $[0,1]$ into sub-intervals. In particular, a group is stable if and only if there exists an interval in this partition that contains the taste parameters of all the group members. This result suggests that if each member has some leverage in choosing her peers, stability occurs when tastes are sufficiently close. ${ }^{5}$ Intuitively, a group is stable only when all its members agree on the optimal way to allocate the group's information gathering across the two issues, and this occurs when tastes are sufficiently similar. The intervals identifying stable groups exhibit interesting comparative statics. Specifically, these intervals are wider for moderate tastes and become narrower as tastes become more extreme. This implies that stability requires more similarity for extreme individuals than for moderate ones.

This comparative statics has strong holding empirically. For example, in recent years there has been an increasing prevalence of online platforms targeted at individuals with extreme tastes (ranging from food aficionados, to gossip followers, to heavy metal music fans). Even mainstream social network sites (such as tribe.net, ning.com, some of the new interfaces under Facebook, etc.) were created to provide channels that allow like-minded individuals to connect more easily.

We also show that as the group size grows large, for non-extreme individuals, stability remains consistent with groups composed of members of different, although sufficiently close, tastes. That is, intermediate intervals in the partition do not converge to singletons. In fact, the only intervals converging to singletons are the two extreme ones. This suggests that, as groups become larger (due to, say, the growth of online communities and forums, or even the initial introduction of email, sms, etc.), heterogeneity persists in groups composed of moderates, but similarity becomes stronger within groups of extremists.

Next, we focus on the case of higher information costs, which is relevant to many environments, ranging from focused wine or book clubs (in which participants have to jointly provide resources, be it money or time), to computer programming forums, whose members decide whether to invest in

\footnotetext{
${ }^{5}$ In fact, new technologies that allow less constrained choices of peers correspond to the emergence of more homogeneous groups. For example, Lynd and Lynd (1929), illustrates how the introduction of the automobile increased the prominence of clubs and coincided with an increase in peer connections based on common interests.
} 
learning a new coding strategy.

When information gathering becomes costly, a free rider problem can arise within groups that are stable absent information costs. Since more extreme individuals have greater incentives to acquire information on the issue they care about, introducing group polarization 'has the benefit of mitigating the free rider problem by weakening the incentive constraints in the information-collection stage.

Our second main result characterizes stable groups for higher information costs. When groups are small, the free rider problem is not severe, and stable groups are the same as those we describe for the low information costs case. As the group size increases, the free rider problem becomes acute and, for sufficiently large groups, stability entails extreme preference polarization. Nonetheless, for intermediate group sizes, two types of groups are stable. First, we show that some of the (sufficiently homogeneous) stable groups in the low information costs case survive as stable. Second, we characterize stable mildly polarized groups. These groups include both some extremists, who collect information on their preferred issue, and some moderates, who are close enough in their preferences to agree on the optimal allocation of the number of signals collected across issues.

Since the driving force behind the appearance of stable heterogeneity is the free rider problem, reducing the cost of information collection plays a similar role to a reduction of group size: they both alleviate the free rider problem. A decrease in the cost of information may occur with the development of a new technology for information gathering (such as the Internet, or a search engine). Thus, our results suggest that, as technology improves, stable groups exhibit more similarity in tastes. Indeed, when information costs are high, polarized individual are necessary in a group to gather information on some issue. As information costs decrease, information gathering becomes feasible for moderate individuals also. Thus, agreement on the optimal way to go about collecting information becomes the prominent criterion around which stable groups form. This is consistent with a large body of empirical work studying the effects of new information technologies on social affiliations. For instance, Sproull and Kiesler (1991) depicted how the introduction of the telephone affected connection between similar individuals. Recently, Rosenblat and Mobius (2004) studied coauthored papers in top economics journals between the years 1969-1999. They showed how the introduction of the Internet in the early 1990 's is linked with a $20 \%$ decrease in the realization of projects with a dissimilar coauthor. 
Our baseline notion of stability is a strong one, in that each individual can potentially deviate to groups composed of agents with any taste combination. This framework fits environments in which the population is very large, such as those pertaining to many online communities and social networks. Nonetheless, in smaller populations, deviations are restricted by the existing partition of agents into groups. We study the case of a small population of agents in Section 5 . We call a partition of the population stable if no agent prefers to join a group (an element in the partition) different than the one she is in or to remain by herself. Certainly, the grand coalition containing all agents is always stable and is the most efficient stable allocation. Moreover, we show that in any stable partition similar agents tend to cluster together. Our last main result provides conditions under which full segregation, a partition into groups that contain only agents with the same taste, can arise as a stable allocation. We exploit the comparative statics developed in the low information cost case to show that, in a similar spirit, segregation is easier to sustain for individuals of extreme tastes than of moderate ones.

To conclude, we make two important remarks to highlight the range of environments our results can be applied to. First, since our analysis is presented in the absence of externalities other than informational ones, it fits most literally social connections having information sharing as a driving force (Internet forums, information networks, blogs, etc.). However, information sharing is one of the motives behind a much wider class of social ties, such as friendships, collaborations, and many others. The framework we present allows us to extract the implications of the information component in the choice of peers. In fact, in the last part of the paper, we discuss how the presence of other externalities, in addition to informational ones, affects our results.

Second, while our model is presented through an information-collection game, its crucial elements are that agents have a limited amount of (possibly costly) effort that they can exert on either issue and that the resulting utilities for all agents exhibit decreasing marginal returns with respect to the total effort exerted on each issue. In fact, in Section 6.1, we show that our results hold for a large class of production functions of public goods. This more general model fits communities that aggregate around the production of some public goods (e.g., freeware development groups, student associations, urban neighborhoods, volunteering groups, and many more). 
Section 6 also illustrates the generality of our results with respect to the number of issues at hand, and the protocols restricting agents' moves from one group to another.

Related Literature. Lazarsfeld and Merton (1954) coined the term homophily - literally meaning "love for the same" - capturing the tendency of socially connected individuals to be similar to one another. ${ }^{6}$ In recent years, there has been a growing body of work identifying homophily across fields, ranging from economics (see Benhabib, Bisin, and Jackson (2010)), to political science (see Huckfeldt and Sprague (1995)), to sociology (see McPherson, Smith-Lovin, and Cook (2001)).

In general, similarity of connected individuals on malleable traits (such as political affiliation, education, etc.) can be rooted in one of two processes: (i) selection, or assortative matching, in which similarity begets association, the process modeled in this paper; or (ii) socialization or convergence, in which social ties generate similarity. One way to disentangle these processes entails a study of exogenous characteristics, such as height or race (see Goeree, McConnell, Mitchell, Tromp, and Yariv (2010), Marmaros and Sacerdote (2006), and Mayer and Puller (2008), who identify significant levels of homophily with respect to these attributes). Another approach is to consider longitudinal data, as in Kandel (1978). She studied adolescent friendships and the extent of similarity across dyadic connections regarding four attributes (frequency of current marijuana use, level of educational aspirations, political orientation, and participation in minor delinquency) at several stages of friendship formation and dissolution. Kandel found that observed homophily was the outcome of a significant combination of both types of processes:

On the theoretical side, several recent papers directly address preferences for similarity. Currarini, Jackson, and Pin (2009) assume homophilic behavior and study its consequences in a friendship formation model.? Peski (2008) derives a preference for similarity endogenously. He assumes certain properties of preferences over friends (complementarities between direct friends and second-degree friends) and the possibility of confusing people who are similar to one another. The necessity to differentiate friends and enemies as much as possible then leads individuals to form friendships with

\footnotetext{
${ }^{6}$ The observation that people connect to those similar to them is, in fact, a rather old one. Aristotle remarked in his Rhetoric and Nichomachean Ethics that people "love those who are like themselves." Plato commented in Phaedrus that "similarity begets friendship."

${ }^{7}$ Bramoullé and Rogers (2010) study a related model of friendship formation in which agents of the same 'group' are more likely to meet (and befriend) one another. They characterize the resulting connection characteristics.
} 
those who are similar.

The underlying idea that the group of players in a strategic situation is, in itself, endogenous motivates some of the work on club formation (see, e.g., Ellickson, Grodal, Scotchmer, and Zame (1999) and Wooders, Cartwright, and Selten (2006)). The basic model of that literature assumes some form of externality across individuals and studies endogenous group formation (often in a general equilibrium setup) in the presence of these externalities. Our approach differs in that externalities in our setting arise only through the sharing of information (specifically, no goods are traded after groups are formed). Furthermore, we focus on the characteristics of the emergent groups (namely, the distribution of tastes as a function of the environment's fundamentals).

Several elements of our model are reminiscent of work in other areas. First, the idea that agents may choose peer groups that match their preferences is an ongoing theme in the theory of public choice, going back to Tiebout (1956). These models define municipalities by the government services and tax rates that they offer. Individuals choose a community that maximizes their utility. Nonetheless, the strategic interaction that follows and the structure of utilities are very different. Furthermore, much of that work is concerned with the efficiency of such processes, rather than with the similarity or heterogeneity of equilibrium communities. ${ }^{8}$ Second, the idea that agents' preferences may alleviate incentive constraints in collective settings with costly information appears in some recent mechanism design literature. ${ }^{9}$ Third, the notion that agents optimally select those with whom they communicate appears also in Calvó-Armengol, De Mạrtí, and Prat (2009). ${ }^{10}$ They consider a set of connected agents who differ in the accuracy of their exogenously provided private information, and are ex-ante identical otherwise. Furthermore, externalities in their model arise through both (costly) information sharing, as well as through ultimate actions that are taken. In contrast, we characterize the endogenous similarity or heterogeneity within 'groups in which all agents freely communicate with all others (and the only externalities present are information-based).

Recently, there has been a proliferation of work illustrating the potential explanatory power of

\footnotetext{
${ }^{8}$ Regarding this line of research, our results in Section 5 share some common elements with Greenberg and Weber (1986) and Demange (1994). We refer to that section for a detailed comparison between our approach and theirs.

${ }^{9}$ See Che and Kartik (2009) and Gerardi and Yariv (2008).

${ }^{10}$ While in our model this choice amounts to selecting agents out of an infinite pool of individuals of all types, in their setting agents choose the intensity of their communication (pertaining to both transmission and reception) with each other agent separately.
} 
social connections regarding individual outcomes across contexts, covering public goods provision, crime, job search, political alliances, trade, friendships, and information collection. ${ }^{11}$ Particularly in view of the vast literature on homophily, an important empirical issue in this literature is that correlations between behavior and outcomes of individuals and their peers may be driven by common unobservables and, therefore, be spurious (see Evans, Oates, and Schwab (1992) and Manski (1993, 2000)). Understanding similarity patterns is potentially important for mitigating such endogeneity problems.

\section{THE MODEL}

There are two issues at stake: $A$ and $B$, each taking a value in $\{0,1\}$. The values of $A$ and $B$ are determined independently at the outset of the game. For expositional simplicity, we assume that each issue $I \in\{A, B\}$ has equal probability of receiving the value 0 or $1 .{ }^{12}$ Issues can stand for many problems, ranging from choices of the best food shop and bookstore, to selecting physicians in two different areas of expertisé (say, a dentist and a pediatrician).

Each agent needs to make a decision on each issue - that is, pick an action $v=\left(v_{A}, v_{B}\right) \in$ $\{0,1\} \times\{0,1\}$. Each agent is characterized by a taste parameter $t \in[0,1]$. The utility of an agent of taste $t$ from choosing $v$ when the realized issues are $A$ and $B$ is given by:

$$
u(t, v ; A, B)=t \mathbf{1}_{A}\left(v_{A}\right)+(1-t) \mathbf{1}_{B}\left(v_{B}\right)
$$

where $1_{I}(\cdot)$ is an indicator function, receiving the value of 1 whenever the argument coincides with issue $I$, and 0 otherwise. Thus, the agent's goal is to match her actions with the realized issues. The taste parameter $t$ measures how much an agent's utility is affected by making the right decision on each issue. ${ }^{13}$ For example, all agents benefit by choosing a superior supermarket and a superior bookstore, but, depending on their consumption patterns, they may differ in how much one affects their utility with respect to the other. Similarly, agents may be affected differently by the selection of

\footnotetext{
${ }^{11}$ This literature is too extensive to survey here. Important work includes Coleman (1966), Conley and Udry (2005), Foster and Rosenzweig (1995), Glaeser, Sacerdote, Scheinkman (1996), Granovetter (1994), Katz and Lazarsfeld (1954), and Topa (2001).

${ }^{12}$ The entire analysis of the paper can be extended directly to asymmetric priors.

${ }^{13}$ While in this setting the issues are common value (so, the right decision for all agents is identical), our analysis would follow the same lines if agents had different opinions on what is the right decision conditional on the realized issue.
} 
an able dentist relative to a pediatrician depending on their age, health, and family status.

Prior to making a decision, each agent can select simultaneously one of two information sources, $\alpha$ or $\beta$, or forego information collection altogether. Information source $\alpha$ provides the realized issue $A$ with probability $q_{\alpha}>0$. That is, upon choosing information source $\alpha$, the agent observes a signal $s \in\{0,1, \varnothing\}$ according to:

$$
\operatorname{Pr}(s=A)=q_{\alpha}, \quad \operatorname{Pr}(s=\varnothing)=1-q_{\alpha} .
$$

Similarly, information source $\beta$ provides the realized issue $B$ with probability $q_{\beta}>0$. In what follows, we sometimes refer to a signal collected from sources $\alpha$ and $\beta$ as " $\alpha$-signal" and " $\beta$-signal," respectively. ${ }^{14}$ The idea behind the assumption that each agent can gather at most one signal is that each agent has a limited budget of resources to allocate to information gathering. ${ }^{15}$ We assume that accessing information is costly: observing a signal of either source comes at a cost of $c \geq 0 .{ }^{16}$ The information collection cost $c$ is strictly positive in any context in which expertise requires effort (e.g., following online reviews, reading specialized magazines, etc.). An agent who decides to forego information collection does not incur any information collection cost.

A group consists of a set of $n \geq 2$ agents. ${ }^{17}$ What defines a group of peers in our model is information sharing. That is, after all information, sources are selected, all signals are realized and made public within the group.

This fully defines a game, the information-collection game. Note that, once information is collected, agents' best response is to follow the signal whenever the realization of an issue is revealed, and to choose any action if the issue is not revealed (all actions leading to the same expected utility). In that sense, for any group composed of agents with tastes $\left(t_{1}, \ldots, t_{n}\right)$, expected payoffs are fully identified by the profile of chosen signals $\left(x_{1}, \ldots, x_{n}\right)$, where $x_{i} \in\{\alpha, \beta, \varnothing\}$ is the source chosen by agent $i$, and

\footnotetext{
${ }^{14}$ Information sources related to one issue can be, for example, a specialized magazine, a TV channel, an Internet search, and so on,

${ }^{15}$ The analysis of the paper does not change if we assume that each agent can acquire any fixed number of signals $h \geq 1$. We return to this generalization in Section 4 .

${ }^{16}$ Note that all types $t \in[0,1]$ face the same cost to gather information on either dimension. Our analysis is robust to the extension in which individuals face a lower cost of accessing information on the dimension they care mostly about.

${ }^{17}$ Assuming that the group size is exogenous captures situations in which agents face a fixed contraint on how much time and effort they can invest in communication, or social interactions. While the group size is exogenous throughout Sections 3 and 4, it becomes endogenous in the analysis in Section 5. In fact, some evidence suggests that there is a cognitive cap on the number of active social ties humans can maintain. Projecting from Primates, Dunbar (1992) estimated this number to be around 150 , while more recent work suggests that the number in modern times is closer to 300 (see, e.g., McCarty, Killworth, Bernard, Johnsen, and Shelley (2000)).
} 
$\varnothing$ stands for agent $i$ not acquiring information.

As a tie-breaking rule, we assume that an agent who is indifferent between acquiring and not acquiring a signal invests in information, and an agent who is indifferent between an $\alpha$ - and a $\beta$-signal chooses an $\alpha$-signal (this simplifies the exposition, but our analysis remains qualitatively intact for any other deterministic tie-brealking rule).

Throughout our analysis, the expected utility of an agent for any given allocation of signals in the group will play a crucial role. Regardless of their action, an uninformed agent makes the right decision on issue $I$ with probability $\frac{1}{2}$. Therefore, when there are $k^{x}$ agents in one's group choosing signal $x$, the probability that an agent (who uses information optimally) makes the right decision on issue $x$ is $1-\frac{1}{2}\left(1-q_{x}\right)^{k^{m}}$, for $x=\alpha, \beta$. Thus, any agent of taste $t^{\prime}$ 's utility when $k^{\alpha}$ and $k^{\beta}$ are the number of signals chosen from source $\alpha$ and $\beta$, respectively, is given by:

$$
U\left(t, k^{\alpha}, k^{\beta}\right) \equiv t\left[1-\frac{1}{2}\left(1-q_{\alpha}\right)^{k^{\alpha}}\right]+(1-t)\left[1-\frac{1}{2}\left(1-q_{\beta}\right)^{k^{\beta}}\right] .
$$

It follows that the information-collection game can be identified with a one-shot complete information game in which each agent's actions are given by the available signals $\{\alpha, \beta, \varnothing\}$ and utilities are specified according to 1 . We focus on equilibria in pure strategies of this induced game. As it turns out, given our tie-breaking rule, a pure equilibrium exists. Throughout the paper, we assume that the most (utilitarian) efficient such equilibrium is selected. As it turns out, there is a simple characterization of an efficient equilibrium.

Lemma 1 (Existence) For any group of $n$ agents with tastes $t_{1} \geq t_{2} \geq \ldots \geq t_{n}$, there exists $\tau^{\alpha} \in\{0, \ldots, n\}$ and $\tau^{\beta} \in\{1, \ldots, n+1\}, \tau^{\beta}>\tau^{\alpha}$, such that all agents $i \leq \tau^{a}$ acquiring the $\alpha-$ signal, all agents $i \geq \tau^{\beta}$ acquiring the $\beta$-signal, and all other agents not acquiring information, constitutes an efficient Nash equilibrium of the information-collection game.

Given a group of $n$ agents with tastes $t_{1} \geq t_{2} \geq \ldots \geq t_{n}$, Lemma 1 allows us to concentrate on equilibria $\left(x_{1}, \ldots, x_{n}\right)$ identified by two thresholds $\tau^{\alpha} \in\{0, \ldots, n\}$ and $\tau^{\beta} \in\{1, \ldots, n+1\}$ such that $x_{1}=\ldots=x_{\tau^{\alpha}}=\alpha$ and $x_{\tau^{\beta}}=\ldots=x_{n}=\beta$ (in particular, if $\tau^{\alpha}=0$ all agents choose a $\beta$-signal, and if $\cdot \tau^{\beta}=n$, all agents choose an $\alpha$-signal). In words, we focus on equilibria in which any agent 
choosing the source $\alpha$ cares more about the issue $A$ than does any agent not choosing any source or choosing the source $\beta$. When $c$ is sufficiently small, the proof of Lemma 1 illustrates that, in fact, any equilibrium of the information-collection game is efficient (so that the number of $\alpha$ and $\beta$ equilibrium signals is determined uniquely).

In the proof of Lemma 1 we show that equilibrium outcomes are ranked according to the volume of information collected on each issue. That is, whenever more $\alpha$-signals are collected in one equilibrium relative to another, more $\beta$-signals will be collected as well in this equilibrium. As a consequence, all agents agree on their most preferred equilibrium outcome. Furthermore, the volume of information collected on each issue corresponding to an efficient equilibrium outcome is uniquely determined.

It is important to note that information collection equilibria may involve agents not collecting information. The fact that information is costly introduces a free rider problem. Indeed, in any equilibrium in which $\tau^{\beta}>\tau^{\alpha}+1$, the agents $i, \tau^{\alpha}<i<\tau^{\beta}$, do not have enough incentives to collect information on either issue. Of course, if $c=0$, the free rider problem disappears and all agents acquire a signal in equilibrium (i.e., $\tau^{\beta}=\tau^{\alpha}+1$ ).

We now consider an extended game composed of two stages. First, each agent of taste $t \in[0,1]$ can choose the tastes of the remaining $n-1$ agents in her group. ${ }^{18}$ Second, the information-collection game described above is played.

Since Lemma 1 guarantees that the number of $\alpha$-signals is determined uniquely in the efficient equilibria of the information-collection game, the agent's optimization problem in the first stage of the extended game is well defined. We denote the set of optimal groups chosen by agent $t$ at the first stage by $O(t)$, each element of which contains $t$ as a member. We define stability in the first stage of the extended game as follows.

Definition (Stable Group) A group $\left(t_{1}, . ., t_{n}\right)^{\prime}$ is stable if it is optimal for all its members - i.e., $\left(t_{1}, . ., t_{n}\right) \in \cap_{i=1}^{n} O\left(t_{i}\right)$

In a stable group, each agent maximizes her expected utility given the tastes of others in the group, foreseeing the equilibrium played in the information-collection game that ensues. This notion

\footnotetext{
${ }^{18}$ For now, we assume that the pool of potential agents to choose from is infinite and that any combination of tastes is feasible. In Section 5 , we restrict the set of agents to a finite population.
} 
of stability is, therefore, a natural equilibrium condition for the group-selection stage in an environment that allows individuals to connẹct in an unconstrained way. For example, online forum users may join any forum that fits their needs. A forum is then stable if all of its active members are satisfied with the set of other active members.

\section{Group Composition}

The goal of this section is to analyze the group properties entailed by the stability notion introduced in Section 2. To do so, we first fix the taste parameter of one agent and identify that agent's optimal peer group choice. We then provide a full characterization of the stable groups.

The following definitions are useful for our analysis.

First, denote by $n^{\alpha}(t)$ the optimal number of $\alpha$-signals the agent with taste parameter $t$ would choose out of a total of $n$ available signals. That is, given $t \in[0,1], n^{\alpha}(t)$ is the maximal integer $k$ such that

$$
U(t, k, n-k) \geq U(t, k-1, n-k+1)
$$

is satisfied. If (2) is not satisfied for any $k$, we define $n^{\alpha}(t)=0$. Let $n^{\beta}(t) \equiv n-n^{\alpha}(t)$. Therefore, $\left(n^{\alpha}(t), n^{\beta}(t)\right)$ represents the unconstrained optimal allocation of $n$ signals for an agent of taste t.

Naturally, $n^{\alpha}(t)$ increases with $t$ and with the group size $n$.

Second, for $x=\alpha, \beta$, let $n_{c}^{x}(t)$ denote the maximal number of $x$-signals acquired in a group for which an agent of taste $t$ is willing to acquire an $x$-signal rather than no signal at all. We term $n_{c}^{x}(t)$ the attainable number of $\alpha$-signals for an individual of taste $t$. Formally, for any $c>0, n_{c}^{\alpha}(t)$ is the maximal integer $h$ such that, no matter how many $w \beta$-signals are acquired,

$$
U(t, h, w)-U(t, h-1, w)=\frac{t}{2}\left(1-q^{\alpha}\right)^{h-1} q^{\alpha} \geq c
$$

Similarly, the attainable number of $\beta$-signals; $n_{c}^{\beta}(t)$, is defined for any $c>0$ as the maximal integer $h$ such that, no matter how many $w \alpha$-signals are acquired,

$$
U(t, w, h)-U(t, w, h-1)=\frac{1-t}{2}\left(1-q^{\beta}\right)^{h-1} q^{\beta} \geq c
$$


When $c=0$, we denote $n_{c}^{\alpha}(t)=n_{c}^{\beta}(t)=\infty$, corresponding to each agent willing to collect a free signal regardless of the number of signals already available.

Next, let $n_{\max }^{\alpha}$ be the maximal attainable number of $\alpha$-signals that corresponds to agents with the most extreme taste parameter $t=1$. That is, $n_{\max }^{\alpha} \equiv n_{c}^{\alpha}(1)$. Analogously, for $\beta$-signals, the maximal attainable number of $\beta$-signals is $n_{\max }^{\beta} \equiv n_{c}^{\beta}(0)$. Note that $n_{c}^{\alpha}(t)$ is increasing in $t$ and $n_{c}^{\beta}(t)$ is decreasing in $t$. Therefore, $n_{\max }^{\alpha}$ and $n_{\max }^{\beta}$ are the maximal number of $\alpha$ - and $\beta$-signals that can be expected to be acquired, respectively, in any equilibrium of the information-collection game.

Finally, let an $A$-extremist be an agent of taste $t$ such that $n_{c}^{\alpha}(t)=n_{\max }^{\alpha}$. In words, $A$-extremists are agents who are willing to acquire the maximal number of $\alpha$-signals. Likewise, $B$-extremists are agents of taste $t$ such that $n_{c}^{\beta}(t)=n_{\max }^{\beta}$. It is easy to see that $A$ - and $B$-extremists are agents with "sufficiently extreme" tastes to be willing to acquire the maximal possible number of signals, and their tastes lie in two intervals of the forms $\left[\underline{t}^{\alpha}, 1\right]$ and $\left[0, \bar{t}^{\beta}\right]$, respectively.

3.1. Optimal Groups. In many environments agents are allowed to choose whom to connect to among a very large populations of individuals of all types. Given a group size $n$, we now analyze the optimal group composition from the point of view of an agent with taste parameter $t \in[0,1]$.

When information is free, any first-best group for the agent with taste parameter $t$ is composed so that $n^{\alpha}(t)$ agents collect an $\alpha$-signal, and $n^{\beta}(t)$ agents collect a $\beta$-signal, therefore achieving the unconstrained optimal allocation for an agent of taste $t$. Groups consisting of all agents sharing the taste parameter $t$ are, therefore, optimal. Nonetheless, since extreme agents of taste $t=1$ (or $t=0$ ) always best respond with the choice of $\alpha$ - (or $\beta$-) signals, an optimal group for the agent of taste $t$ can also be composed of just the right number of extremists on each side, thereby achieving maximal polarization.

When information is costly (i.e., $c>0$ ), in choosing a group, an individual has to consider her peers' incentives to acquire information (not only their choice of informational source). In particular, the number of $x$-signals cannot exceed $n_{\max }^{x}$ for any source $x=\alpha, \beta$. Therefore; the agent of taste parameter $t$ has hope of achieving her unconstrained optimum only if $n^{x}(t) \leq n_{\max }^{x}$ for $x=\alpha, \beta$.

The following Proposition describes the optimal groups. 
Proposition 1 (Optimal Groups) Consider an agent of taste parameter $t$. In any optimal group, the agent

1. achieves the unconstrained optimal allocation $\left(n^{\alpha}(t), n^{\beta}(t)\right)$ if and only if it is feasible, i.e., $n^{x}(t) \leq n_{\max }^{x}$ for $x=\alpha, \beta$, and it induces the agent to invest in information, i.e., $n^{x}(t) \leq n_{c}^{x}(t)$ for at least one $x \in\{\alpha, \beta\}$

2. implements at most $n_{\max }^{x}$ signals $x$ for each $x=\alpha, \beta$ for which $n^{x}(t)>n_{\max }^{x}$.

In particular, if information is free $(c=0)$, any optimal group achieves the unconstrained optimal allocation. Such optimal groups must involve $n^{\alpha}(t)$ agents who care enough about issue $A$ so that they choose an $\alpha$-signal in the information-collection game, and $n^{\beta}=n-n^{\alpha}(t)$ agents who care enough about issue $B$ to choose a $\beta$-signal in the information-collection game. Thus, optimal groups must be characterized by two thresholds - one assuring that $n^{\alpha}(t)$ agents have a sufficiently high taste parameter, and the other assuring that $n^{\beta}(t)$ agents have a sufficiently low taste parameter.

Therefore, when information is free, in situations in which one agent has leverage in choosing her group partners, the agent cares only'about order-statistic type of attributes of the group's distribution of tastes. Therefore, optimal groups can entail maximal polarization, containing extreme agents on both sides of the taste spectrum.

When information is costly $(c>0)$, the pressure to choose more extreme individuals is even more pronounced as these are the agents with the highest willingness to acquire information. In other words, the presence of the free rider problem may induce an agent to select more polarized sets of peers.

3.2. Stable Groups. In the previous section we described the optimal group choice for any individual of a given taste $t$. However, in most applications, all members of a group of peers have some leverage in choosing the other members. The goal of this section is to characterize stable groups, which are optimal for all of their members. For illustration purposes, we first characterize the stable groups for very low costs of information gathering (or free information). We then expand the analysis to arbitrairy information costs, showing how the structure of stable groups changes as the information gathering cost $c$ increases. 


\subsubsection{Low Information Costs}

As discussed above, if information is free, an agent's optimal group entails her unconstrained optimal allocation of $n$ signals across the sources $\alpha$ and $\beta$ being selected in the information-collection game. Thus, in a stable group in which all agents optimize on their peers' tastes, all agents have to agree on the optimal allocation of signals across the two sources. In particular, a group formed by identical agents is always stable and stable groups always exist in this setting. More generally, when information is free, stable groups are characterized as follows.

Proposition 2 (Free Information - Stable Groups) For any group size n, there exists a partition $\left\{T_{k}^{n}\right\}_{k=0}^{n}$ of the interval $[0,1]$, where $T_{0}^{n}=[0, t(1)), T_{k}^{n}=[t(k), t(k+1))$, for $k=1, \ldots, n-1$, and $T_{n}^{n}=[t(n), 1]$ such that:

1. A group comprised of agents with tastes $\left(t_{1}, \ldots, t_{n}\right)$ is stable if and only if there exists $k=$ $0, \ldots, n$, such that for all $i, t_{i} \in T_{k}^{n}$. That is, all taste parameters in the group belong to the same element of the partition.

2. The length of the intervals $\left\{T_{k}^{n}\right\}_{k=1}^{n-1}$ is increasing for $k=1, . . \widehat{k}$ and decreasing for $k=$ $\widehat{k}, . ., n-1$, where $\widehat{k}$ is such that $\frac{1}{2} \in T_{\widehat{k}}^{n}$ or $\frac{1}{2} \in T_{\widehat{k}+1}^{n}$. Thus, the intervals $\left\{T_{k}^{n}\right\}_{k=1}^{n-1}$ are narrower for extreme tastes, and wider for moderate tastes. ${ }^{19}$

While Proposition 1 shows that, from an individual point of view, optimal groups can entail extreme polarization, the first part of Proposition 2 guarantees that stable groups can be formed only by agents whose tastes are close enough - namely, they lie in one of the intervals $T_{k}^{n}$.

Furthermore, the length of the intervals $\left\{T_{k}^{n}\right\}_{k=0}^{n}$ provides a proxy for equilibrium homophily: the narrower an interval is, the closer the agents' tastes have to be in order for them to form a stable group. The second part of Proposition 2 addresses how the intervals' lengths are affected by the proximity of the intervals to the extreme tastes. In particular, point (2) of Proposition 2 implies that stability requires more similarity for extreme individuals than for moderate ones.

As we mention in the Introduction, this comparative statics provides insights into the recent popularity of online platforms targeted at individuals with extreme tastes (e.g., food aficionados,

\footnotetext{
${ }^{19}$ In the Appendix, we show that, under mild conditions on $q_{\alpha}, q_{\beta}$, and $n$, this result extends to the entire sequence $\left\{T_{k}^{n}\right\}_{k=0}^{n}$. For example, $\left(1-q_{\alpha}\right)\left(1-q_{\beta}\right)<1 / 2$ and $n$ high enough, are sufficient to guarantee this.
} 


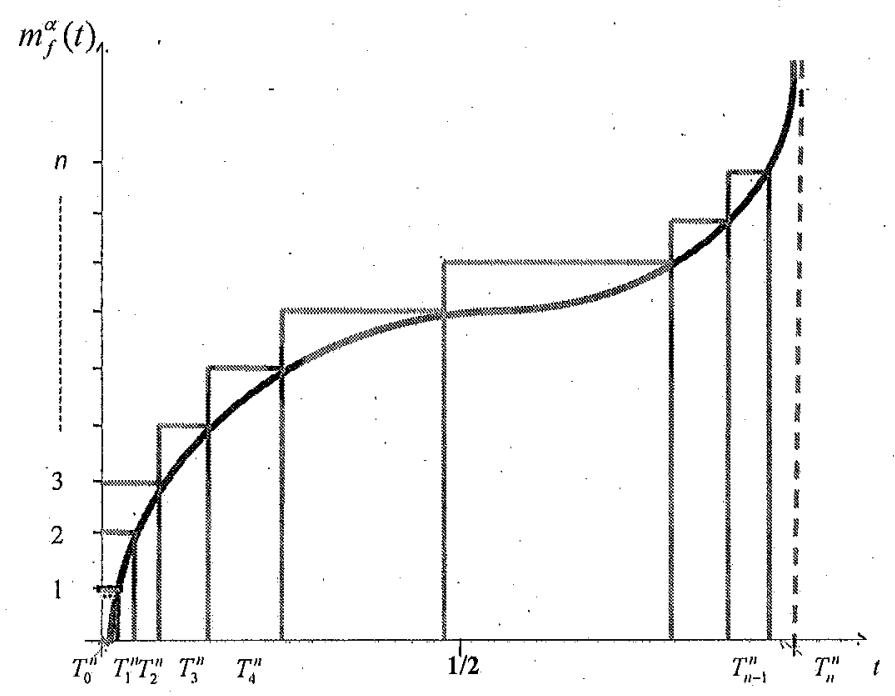

Figure 1: Stable Groups in the Free Information Case

gossip followers, heavy metal music fans, etc.). To exploit this trend, some of the mainstream social network sites (such as tribe.net, ning.com, and some of the new interfaces under Facebook) have taken action to facilitate connections between like-minded individuals.

In order to see the intuition underlying these results, it is useful to consider the optimal number of $\alpha$-signals $n^{\alpha}(t)$ that each agent of taste $t$ would choose. Indeed, denoting by $m^{\alpha}(t)$ the real number achieving equality within constraint (2), we get that $n^{\alpha}(t)=\left\lfloor m^{\alpha}(t)\right\rfloor$ if $m^{\alpha}(t) \in[0, n], n^{\alpha}(t)=0$ if $m^{\alpha}(t)<0$, and $n^{\alpha}(t)=n$ if $m^{\alpha}(t)>n$. Roughly speaking, $m^{\alpha}(t)$ captures the point at which an agent of taste $t$ equates the marginal return from an $\alpha$-signal with the marginal return from a $\beta$-signal. The function $m^{\alpha}(t)$ is depicted in Figure 1.

Each interval in the partition $\left\{T_{k}^{n}\right\}_{k=0}^{n}$ includes all taste parameters of agents who agree on a given optimal allocation of signals. In Figure 1, agreement on the number of $\alpha$-signals $n^{\alpha}(t)=k$ corresponds to the interval of taste parameters that is projected from $[k, k+1)$ on the $y$-axis. That is, the interval of tastes for which $m^{\alpha}(t) \in[k, k+1)$ and $n^{\alpha}(t)=k$.

Let us turn to points (1) and (2) of Proposition 2. First, as $t$.increases, an agent with taste parameter $t$ cares more about issue $A$ and so that agent's optimal allocation entails more $\alpha$-signals. 
As a result, $m^{\alpha}(t)$ is strictly increasing. The monotonicity of $m^{\alpha}(t)$ guarantees the sorting result in the first part of Proposition 2: the set of taste parameters agreeing on the optimal allocation of signals must be an interval.

Second, note that an agent with taste parameter $t=\frac{1}{2}$ cares equally about both issues. In particular; by raising $t$ above $\frac{1}{2}$, the relative value of an $\alpha$-signal increases since the agent cares more about issue $A$ than issue $B$. However, a countervailing force is in effect. Indeed, when the optimal number of $\alpha$-signals increases with $t$ (and the optimal number of $\beta$-signals decreases), the marginal return of an $\alpha$-signal relative to a $\beta$-signal decreases. For the salke of simple intuition, let us focus on the symmetric case $q_{\alpha}=q_{\beta} \equiv q$. The optimal number of $\alpha$-signals for an agent of type $t>1 / 2$ is approximately $m^{\alpha}(t)$ (in precise terms, it is the integer part of $m^{\alpha}(t)$ ). That is,

$$
\frac{t}{1-t}=\frac{q_{\beta}\left(1-q_{\beta}\right)^{n-m^{\alpha}(t)}}{q_{\alpha}\left(1-q_{\alpha}\right)^{m^{\alpha}(t)-1}}=(1-q)^{n-2 m^{\alpha}(t)+1} \text {. }
$$

Both sides of condition (5) are increasing in $t$, the left hand side capturing the first, direct effect of an increase in $t$, and the right hand side representing the marginal return of a $\beta$-signal relative to an $\alpha$-signal.

Defining the function corresponding to the right hand side of condition (5) as $M(k) \equiv(1-q)^{n-2 k+1}$, it is easy to see that $\frac{M(k+1)}{M(k)}$ is equal to a constant, namely, $(1-q)^{-2}$. Thus, the relative marginal returns of an $\alpha$-signal decrease (i.e., $M(k)$ increases) at a constant rate. On the other hand, the left hand side of (5) increases at rapidly increasing rates when $t>1 / 2$. This implies that, as $t$ increases, $m^{\alpha}(t)$ has to increase at increasing rates to satisfy (5). In other words, $m^{\alpha}(t)$ must be convex for $t>1 / 2$ and concave for $t<1 / 2$. This induced shape of $m^{\alpha}(t)$ implies the property described in point (2) of Proposition 2 directly. ${ }^{20,21}$

Notice that for sufficiently low costs, all agents are willing to acquire any signal regarcless of the

\footnotetext{
${ }^{20}$ The reason why the extreme intervals can generally follow a different pattern is the following. Note that the extreme intervals collect all the taste parameters $t$ such that the problem of the optimal allocation of signals across sources $\alpha$ and $\beta$ has a corner solution. Then, for example, for a given $q_{\beta}$, if both $q_{\alpha}$ and $n$ are very low, there is a wide range of $t$ 's for which it is optimal to get $n \beta$-signals (the marginal returns from source $\alpha$ are very low). In order for the pattern to carry through for the extreme intervals, the signal accuracies and the group size need to be sufficiently large.

${ }^{21}$ These intuitions are generalized in Section 6.1. While here we assume a particular signal generation process, as long as the information technology is such that the optimal number of signals on one issue is monotonic in the type parameter, stable groups exhibit sorting as in point (1) of Proposition 2. When the marginal return of a signal does not decrease "too quickly," the comparative statics described in point (2) of Proposition 2 holds.
} 
allocation of signals in their group (formally, $n_{c}^{x}(t) \geq n$ for all $t, x=\alpha, \beta$ ). Therefore, for sufficiently. low costs the characterization of stable groups is precisely that of Proposition 2.

From an empirical point of view, these results are important for understanding the link between group composition and extremism of opinions. Some of the recent social psychology literature (see Myers (2007)) suggests that more homogeneous groups tend to exhibit far more extreme opinions than heterogeneous ones following group interactions. While the social psychology literature focuses on mechanisms by which group dynamics generates extremism, our results imply that it is important to account for the way these groups are created to begin with. Specifically, the comparative statics we identify indicate that more extreme individuals would be more likely to form homogeneous groups in the first place.

To conclude, it is interesting to study how the similarity requirements for stability (i.e., the length distribution of the intervals) depend on the accuracies of the signals. Intuitively, when signal accuracies are both very high, the marginal return from an additional signal when $n / 2 \alpha$-signals and $n / 2 \beta$ signals have been collected (corresponding to the largest middle interval) is very low. In particular, the marginal return from an additional $\alpha$-signal is not that different from the marginal return from an additional $\beta$-signal and so the class of types who find the allocation $(n / 2, n / 2)$ optimal is rather large. In other words, as accuracies increase, the size of the large middle interval increases, and stability is consistent with more ingroup heterogeneity.

\subsubsection{Costly Information}

We now turn to the characterization of stable groups when information entails non-trivial costs. First of all, note that stable groups always exist. Indeed, having $n$ agents of taste $t=1$ and $\min \left\{n, n_{\max }^{\alpha}\right\}$ all acquiring an $\alpha$-signal, or analogously, having $n$ agents of taste $t=0$ and $\min \left\{n, n_{\max }^{\beta}\right\}$ all acquiring a $\beta$-signal, both constitute stable groups. ${ }^{22}$ Our' goal is to characterize all stable groups in the presence of information costs. We show that the structure of stable groups depends crucially on the group's size $n$.

While we present the analysis for different group sizes, our results have a natural analogue.in terms

\footnotetext{
${ }^{22}$ We avoid issues of equilibrium selection in that we assume that when a subgroup of agents of identical tastes acquires information, they cannot guarantee lower investment in information by shifting to a different group in which a different agent acquires their specific signals.
} 
of information gathering costs: increasing the size of the group is tantamount to increasing costs in that both make the free rider problem more severe. Formally, note that by increasing $c$, we reduce the number of attainable signais $n_{c}^{x}(t)$ for all $t, x=\alpha, \beta$ (in particular, we lower $n_{\max }^{\alpha}$ and $n_{\max }^{\beta}$ ). Thus, our results shed light on the effects of new technologies that decrease costs of information gathering (e.g., the introduction of the telephone, Internet, search engines, etc.). Similarly, they tie to periods of time in which media spread was experienced, effectively decreasing the costs of information acquisition.

Large Groups. Given the definition of $n_{\max }^{\alpha}$ and $n_{\max }^{\beta}$, the maximal number of attainable signals that can conceivably be acquired in equilibrium is $n_{\max }^{\alpha}+n_{\max }^{\beta}$. In that respect, we refer to groups containing more than $n_{\max }^{\alpha}+n_{\max }^{\beta}$ members as large. The following proposition provides the characterization of large stable groups.

Proposition 3A (Stability - Large $n$ ) When $n>n_{\max }^{\alpha}+n_{\max }^{\beta}$, stable groups take one of the following forms:

1. $n_{\max }^{\alpha} A$-extremists and $n_{\max }^{\beta} B$-extremists;

2. $n$ agents with taste $t=1$, or $n$ agents with taste $t=0$.

The first part of Proposition 3A describes stable groups in which the number of signals gathered in the information-collection phase is maximized. When groups are very large, any agent with $t \in(0,1)$ would desire a group in which the maximal amount of information on both issues is acquired. The only way to achieve this volume of information is to have a group in which at least $n_{\max }^{\alpha}$ agents are $A$-extremists and at least $n_{\max }^{\beta}$ agents are $B$-extremists. Note that, while in the free-information case stability always entailed some degree of similarity, the first part of Proposition 3A suggests that for large group sizes, stability is consistent with extreme group polarization. ${ }^{23}$

In the second part of Proposition 3A we describe groups that are always stable, in which all agents have the same, extreme, taste parameter. This is a knife-edge case in which all agents get no utility from signals on the issue they do not care about. In such groups, the maximal attainable number of signals is collected on the issue all of the members do care about.

\footnotetext{
${ }^{23}$ We refer to groups that contain at least one $A$-extremist and one $B$-extremist as extremely polarized. In fact, Proposition $3 \mathrm{~A}$ suggests that as $n$ increases (or $c$ decreases) there will be a greater volume of agents on each side of the taste spectrum. These groups are stable in the free-information case only when all agents agree on the optimal allocation of signals.
} 
Going back to the analogy between large group size $n$ and high information cost $c$, as noted, a higher information cost $c$ lowers both $n_{\max }^{\alpha}$ and $n_{\max }^{\beta}$. Thus, Proposition $3 \mathrm{~A}$ suggests that high costs induce extreme polarization.

Small Groups. In general, the optimal composition of signals for each agent entails the collection of signals from both sources. When groups are small, the free rider problem is weaker, implying the possibility of the unconstrained optimal allocation being consistent with stability.

The following Lemma will be useful in linking stable groups with costly information to those identified in the free-information case through Proposition 2. For any $t \in[0,1]$, we define $n_{c}(t) \equiv$ $n_{c}^{\alpha}(t)+n_{c}^{\beta}(t)$ as the total attainable number of signals for an agent of taste $t$. It is the maximal number of signals that a group of agents of the same taste $t$ would be willing to acquire in equilibrium.

Lemma 2 (Individual Incentives and Group Size) Whenever $n \leq n_{c}(t), n^{x}(t) \leq n_{c}^{x}(t)$ for $x=$ $\alpha, \beta$.

Lemma 2 links the size of the group and the personal incentives to acquire information of an individual of taste $t$. As long as group size is sufficiently small (relative to the total attainable number of signals for an agent of taste $t$ ), the agent engages in information acquisition when her unconstrained optimal allocation of signals is instated. To see why this is the case, suppose that $n^{\alpha}(t)+n^{\beta}(t)=n \leq n_{c}(t)$ but, for instance, $n^{\alpha}(t)>n_{c}^{\alpha}(t)$. Then, it must be the case that $n^{\beta}(t)<n_{c}^{\beta}(t)$. Since $n^{\alpha}(t)$ and $n^{\beta}(t)$ represent the unconstrained optimal allocation, they are selected in a way that (approximately) equates the marginal returns from signals on either source. However, since $n^{\beta}(t)<n_{c}^{\beta}(t)$, the marginal benefit from the $n^{\beta}(t)$-th $\beta$-signal is greater than $c$. Thus, the marginal benefit from the $n^{\alpha}(t)$-th $\alpha$-signal should be greater than $c$ as well, in contradiction to $n^{\alpha}(t)>n_{c}^{\alpha}(t)$.

We now turn to the characterization of stable groups when $n<n_{\max }^{\alpha}+n_{\max }^{\beta}$. First, some of the homogeneous groups described in Proposition 2 are still stable when information is costly. Denote by $m_{c}^{\alpha}(t)$ and $m_{c}^{\beta}(t)$ the real numbers achieving equality within the constraints (3) and (4), respectively. For $x=\alpha, \beta, n_{c}^{x}(t)=\left\lfloor m_{c}^{x}(t)\right\rfloor$ if $m_{c}^{x}(t) \geq 0$ and $n_{c}^{x}(t)=0$ if $m_{c}^{x}(t)<0$ (by construction, $m_{c}^{x}(t)<$ $n_{\max }^{x}+1$ for $\left.x=\alpha, \beta\right)$. The number $m_{c}^{x}(t)$ captures the point at which an agent of taste $t$ equates the marginal return from an $x$-signal to the cost $c$. The total attainable number of signals for an agent 


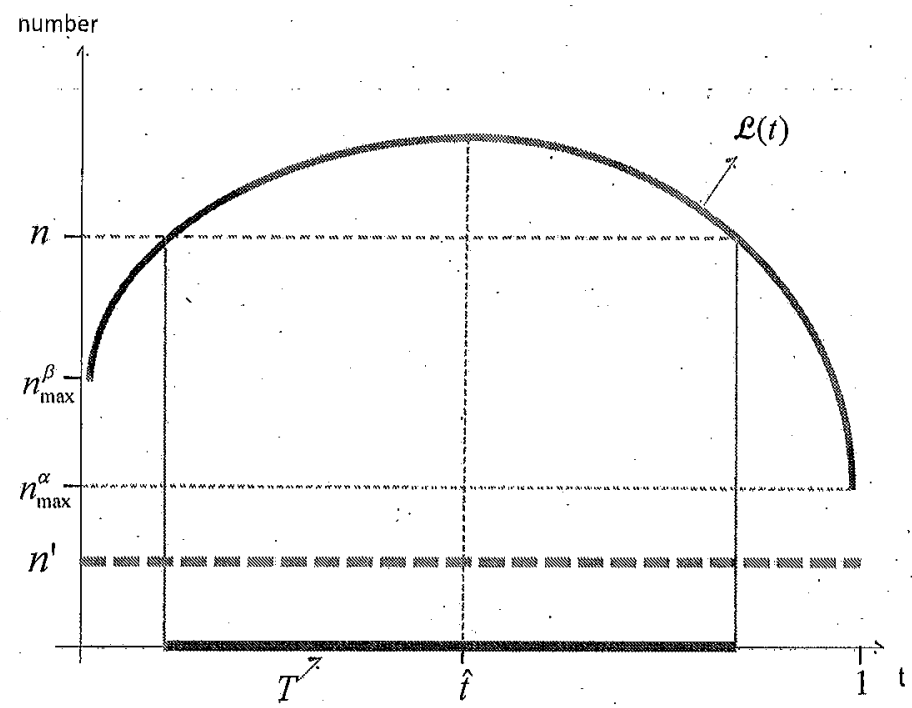

Figure 2: Small Group Size (Low Costs)

of taste $t$, or $n_{c}(t)$, is then approximated by the function $\mathcal{L}(t)=\max \left\{m_{c}^{\alpha}(t), 0\right\}+\max \left\{m_{c}^{\beta}(t), 0\right\}$ depicted in Figure $2 .{ }^{24}$ It is easy to see that $\mathcal{L}(0)=n_{\max }^{\beta}, \mathcal{L}(1)=n_{\max }^{\alpha}$, and, as we show in the Appendix, $\mathcal{L}(t)$ is piece-wise concave and reaches a maximum at $\hat{t} \in(0,1)$ when it is concave and positive. $^{25}$

Looking at Figure 2; when $\min \left\{n_{\max }^{\alpha}, n_{\max }^{\beta}\right\}<\dot{n} \leq n_{c}(\hat{t})$, there is always a set of taste parameters $T$ such that for any $t \in T, n \leq n_{c}(t)$. By Lemma 2 , this guarantees that groups formed by agents sharing the same unconstrained optimal allocation (corresponding to stable groups in the free-information case) and with tastes within $T$, are able to achieve their optimal allocation in the costly-information case as well. That is, if stable groups of size $n$ in the free-information case are characterized by the partition $\left\{T_{k}^{n}\right\}_{k=0}^{n}$, when information comes at a cost of $c$, for all the elements in the partition such that $T_{k}^{n} \cap T \neq \varnothing$, any group of $n$ agents in $T_{k}^{n} \cap T$ is stable.

We now focus our attention on polarized groups. The extremely polarized groups appearing for large groups (Proposition 3A) are not stable for smaller group sizes. ${ }^{26}$ Nonetheless, for interme-

\footnotetext{
${ }^{24}$ The shape portrayed in Figure 2 corresponds to sufficiently low costs. See discussion below regarding comparative statics with respect to $c$ for a description of the dependence of $\mathcal{L}(t)$ on $c$.

${ }^{25}$ The concavity of $\mathcal{L}(t)$ is a direct consequence of the decreasing marginal returns from signals.

${ }^{26}$ To see why, consider a polarized group in which some agents are $A$-extremists (and their optimal groups entail
} 
diate group sizes, ones for which information on at least one dimension can be maximized (i.e., $\left.n>\min \left\{n_{\max }^{\alpha}, n_{\max }^{\beta}\right\}\right)$, mildly polarized groups in which some agents have extreme tastes, while others have moderate (and similar) tastes, can be stable. Indeed, suppose that $n>n_{\max }^{\alpha}$. We now illustrate that a stable group can be comprised of a sub-group of $A$-extremists that get $n_{\max }^{\alpha} \alpha$-signals, and a sub-group of moderates, who all acquire $\beta$-signals. Formally, assume that $n_{\max }^{\alpha}$ agents care sufficiently about issue $A$ so that: (i) they are $A$-extremists (i.e., they have strong enough incentives to collect $n_{\max }^{\alpha} \alpha$-signals); and (ii) their unconstrained optimal allocation involves at least $n_{\max }^{\alpha} \alpha$-signals (so that they do not prefer groups with greater $\beta$-signal acquisition). To capture these restrictions, we define for $x=\alpha, \beta$ :

$$
W^{x}=\left\{t \mid n_{c}^{x}(t)=n_{\max }^{x} \text { and } n^{x}(t) \geq n_{\max }^{x}\right\}
$$

It is easy to see that $W^{\alpha}$ and $W^{\beta}$ are intervals of the form $W^{\alpha}=[\underline{t}, 1]$ and $W^{\beta}=[0, \dot{\bar{t}}]$. Suppose $n_{\max }^{\alpha}$ of the $n$ agents have taste parameters in the interval $W^{a}$. By construction, these agents are in an optimal group whenever the number of $\alpha$ - and $\beta$-signals collected is $n_{\max }^{\alpha}$ and $n-n_{\max }^{\alpha}$, respectively,

We choose the remaining $n-n_{\max }^{\alpha}$ agents to satisfy two conditions: (i) they care enough about issue $B$ to have sufficient incentives to collect $n-n_{\max }^{\alpha} \beta$-signals; and (ii) they care enough about issue $A$ so that they would not prefer a group in which more than $n-n_{\max }^{\alpha} \beta$-signals are collected. Formally, for $x, y=\alpha, \beta, x \neq y$, we define:

$$
Z^{x}=\left\{t \mid n_{c}^{x}(t) \geq n^{x}(t) \text { and } n^{x}(t)=n-n_{\max }^{y}\right\}
$$

Thus, if we select the remaining $n-n_{\max }^{\alpha}$ agents to have taste parameters within $Z^{\beta}$, by construction, these agents will be in an optimal group. In particular, combined with the $n_{\max }^{\alpha}$ agents with tastes in $W^{\alpha}$, they form a stable group. Note that for any taste parameter $t$ in $Z^{\beta}, n^{\beta}(t)=n-n_{\max }^{\alpha}<n_{\max }^{\beta}$. Therefore, $Z^{\beta}$ does not contain the extreme taste parameter $t=0$. This implies that the stable groups we just constructed involve a milder degree of polarization with respect to the large-group-size case.

The following proposition summarizes our discussion and provides the full characterization of stable groups for small group sizes (where we continue using the notation of $\left\{T_{k}^{n}\right\}_{k=0}^{n}$ for the partition

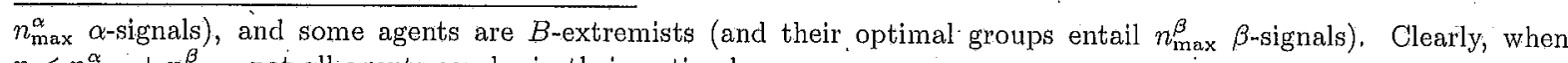
$n<n_{\max }^{\alpha}+n_{\max }^{\beta}$, not all agents can be in their optimal group. 
corresponding to stable groups in the free-information case) ${ }^{27}$

Proposition 3B (Stability - Small $n$ ) When $n<n_{\max }^{\alpha}+n_{\max }^{\beta^{-}}$, stable groups take one of the following forms:

1. If there is an interval $T$ such that $n \leq n_{c}(t)$ for all $t \in T$, then, for any $k=0, \ldots, n$ for which $T \cap T_{k}^{n} \neq \varnothing$, a homogeneous group comprised of any $n$ agents of tastes in $T \cap T_{k}^{n}$.

2. If $n>n_{\max }^{x}$, a mildly polarized group comprised of $n_{\max }^{x}$ agents with tastes in $W^{x}$, and $n-n_{\max }^{x}$ agents with tastes in $Z^{y}$.

3. $n$ agents of taste $t=1$, or $n$ agents of taste $t=0$.

Regarding the case $n=n_{\max }^{\alpha}+n_{\max }^{\beta}$, whenever $n_{\max }^{x}>1$ for $x=\alpha, \beta$, the groups described in Proposition $3 \mathrm{~A}$ are the only stable ones. However, if $n_{\max }^{x}=1$ for some $x$, say $\alpha$, the full characterization of the stable groups includes additional types of groups consisting of $n_{\max }^{\beta}$ agents on the $t=0$ extreme and one moderate agent. Since the full characterization involves some minor technical subtleties without adding qualitative novelties, we refer the interested reader to Proposition $3 \mathrm{C}$ presented. in the Appendix. ${ }^{28}$

Note that a consequence of our results is that there is a set of moderate taste parameters such that individuals with those tastes can only be part of stable groups that are either small (emulating the free-information environment) or very large (in which the moderate agents free ride on the extremists in the group, who collect all the information).

The analogy between small group size $n$ and small information cost $c$ suggests that, as $c$ decreases, some polarization can still persist in stable groups (point (2) of Proposition 3B), but it is milder

\footnotetext{
${ }^{27}$ Note that whenever $n<\min \left\{n_{\max }^{\alpha}, n_{\max }^{\beta}\right\}$ part (3) of Proposition $3 \mathrm{~B}$ is subsumed in part (1).

${ }^{28}$ It is interesting to consider the consequences of side payments. When groups are small, so that stability entails the acquisition of the same signal profile of the free-information case, side payments have no consequence. For large groups, side payments allow agents to share the cost of information and invest (albeit indirectly) in more than one signal. In that case, the availability of side payments gerierates more information acquisition. In particular, for large group sizes, the introduction of side paymerit will tend to generate more similarity in stable groups. To see why, consider a polarized group (i.e, a group formed only by extemists on both issues). In such a group, an extremist on issue $A$ will have an incentive to pay an extremist on issue $B$ to acquire an $\alpha$-signal rather than a $\beta$-signal. However, the same goal could have been achieved at a lower cost by selecting another extremist on issue $A$ as a peer in the first place. This suggests that extreme polarization still arises in groups that are suffciently large (but the lower bound on group size for extreme polarization to arise is higher than in our setting).
} 


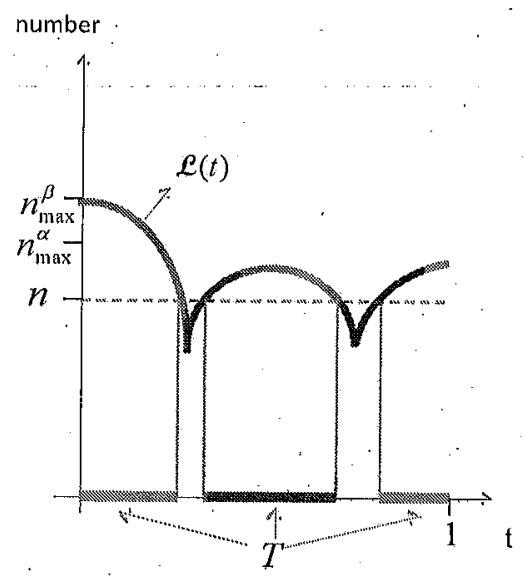

(a)

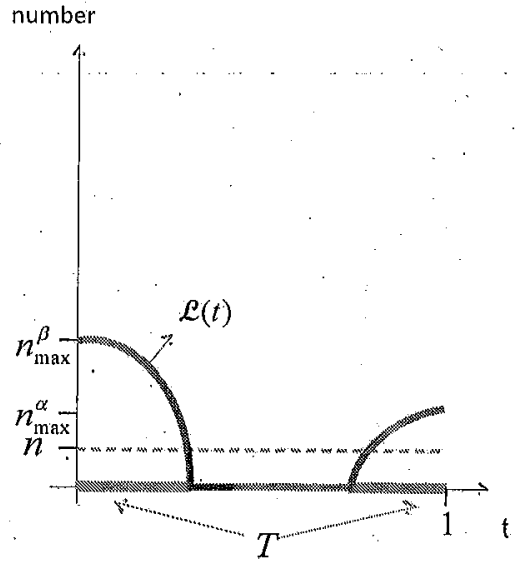

(b)

Figure 3: Stable Groups as Costs Increase

than the extreme polarization emerging for high $c$. Eventually, for sufficiently low $c$, polarized groups disappear. Moreover, as $c$ decreases, homogeneous groups identical to those identified as stable in the free-information case emerge as stable (point (1) of Proposition 3B).

A message that comes out of our analysis is that when information gathering becomes cheaper (as a result, for instance, of better information technologies), stable groups tend to become more homogeneous. As mentioned in the Introduction, this insight is backed up by a large body of empirical work. For example, the introduction of the telephone made social affiliations depend far more on shared interests (e.g., Sproull and Kiesler (1991)). Similarly, the introduction of the Internet is associated with a significant increase in the similarity of academic coauthors (see Rosenblat and Mobius (2004) and references therein).

The Effects of Costs on Homogeneous Groups. Information costs affect the total attainable signals and then, via Lemma 2 and point (1) of Proposition 3B, the interplay between group size and the selection of stable homogeneous groups from the free-information case. When costs are low, any agent is willing to acquire at least one signal on either issue (i.e., for all $t, n_{c}^{\alpha}(t), n_{c}^{\beta}(t) \geq 1$ ). In that case, $\mathcal{L}(t)$ takes the form depicted in Figure 2. In terms of the stable homogeneous groups, as 
long as $n \leq \min \left\{n_{\max }^{\alpha}, n_{\max }^{\beta}\right\}$ (for instance, $n^{\prime}$ in Figure 2), any stable group in the free-information environment is also stable when information comes at a cost $c$. As $n$. increases, the set $T$ shrinks and contains less extreme taste parameters (for instance, $n$ in Figure 2). Thus, when costs are low, as. group size increases, the homogeneous stable groups that survive are those composed of members with more moderate tastes.

As costs increase, extreme agents are willing to acquire information only on the issue they care most about, and $\mathcal{L}(t)$ becomes piece-wise concave (see panel (a) of Figure 3). For such situations, homogenous stable groups are composed of members with either moderate or extreme tastes.

As costs increase even more, there may be a range of moderate types who are not willing to acquire information on either issue and $\mathcal{L}(t)$ has a dip for a range of moderate tastes (see panel (b) of Figure 3). In that case, whenever $n \leq \mathcal{L}(t)$ for some $t$, either $n_{c}^{\alpha}(t)=0$ or $n_{c}^{\beta}(t)=0$. Suppose $n_{c}^{\alpha}(t)=0$. By construction, $n^{\beta}(t) \leq n_{c}^{\beta}(t)$. From Lemma 2, it must then be the case that $n^{\alpha}(t) \leq n_{c}^{\alpha}(t)=0$. In particular, $t$ belongs to the extreme interval $T_{0}^{n}$. An analogous analysis pertains to the case in which $n_{c}^{\beta}(t)=0$. We therefore deduce that when costs are high, and $n$ is sufficiently low, the homogenous stable groups, correspond to extreme stable groups in the free-information case.

\section{Group Size}

Many recent technologies, such as e-mail, instant messaging, online networks, etc., allow individuals to connect to one another with greater ease. It has been empirically observed that larger groups tend to be characterized by an increased degree of similarity. ${ }^{29}$ In light of this evidence, we now look at how the results in Section 3.2 are affected by arbitrarily increasing group size.

First of all, note that for any $c .>0$, we can identify the maximal number of attainable signals on both issues, $n_{\max }^{\alpha}+n_{\max }^{\beta}$. Thus, for any group size $n^{\prime}>n_{\max }^{\alpha}+n_{\max }^{\beta}$, the only stable groups are the ones characterized in Proposition 3A, with an arbitrarily large number of agents free riding on the information collected by $n_{\max }^{\alpha} A$-extremists and $n_{\max }^{\beta} B$-extremists.

Therefore, we now focus our attention on the free-information case $c=0$. Although this is a knife-edge case, it applies to a wide range of examples in which some form of information collection is par for the course - e.g., which Internet forum or online social network to join, which part of the

\footnotetext{
${ }^{29}$ See, for instance, Currarini, Jackson; and Pin (2009) and references therein.
} 
newspaper to read in the morning, and so on.

As before, we denote by $\left\{T_{k}^{n}\right\}_{k=0}^{n}$ the partition of the unit interval into sub-intervals defining stability. We call the stable groups that choose all signals from the same source (i.e., have taste parameters in either $T_{0}^{n}$ or in $T_{n}^{n}$ ) extreme stable groups. We call all other stable groups non-extreme stable groups.

Proposition 4 (Free Information-Large $n$ ). For any two agents of taste parameters $t, t^{\prime}$ :

1. If they both belong to a non-extreme stable group of size $n \geq 2$, then they both belong to a non-extreme stable group of some size $n^{\prime}>n$.

2. If they both belong to an extreme stable group of size $n$, then they both belong to an extreme stable group of any smaller size $n^{\prime}<n$. Furthermore, extreme stable groups become fully homogeneous (containing only the most extreme agents) as group size becomes infinitely large.

Since all agents with taste parameters belonging to the same interval $T_{k}^{n}$ have to agree on how to optimally allocate $n$ signals across the $\alpha$ - and $\beta$-sources, one may conjecture that, as $n$ grows arbitrarily large, these intervals converge to singletons, larger groups displaying greater homophily. Proposition 4 illustrates that this is the case only for extreme stable groups. Nonetheless, for non-extreme stable groups, Proposition 4 shows that this conjecture does not hold, and the same degree of similarity found for small group sizes tends to persist for larger sizes as well. In particular, the first part of Proposition 4 guarantees that, as the group size increases, the non-extreme intervals. $\left\{T_{k}^{n}\right\}_{k=1}^{n-1}$ do not converge to singletons. It is sufficient for two different agents to agree on an optimal source allocation for a given group size $n \geq 2$ for them to keep on agreeing on an allocation for larger and larger groups. ${ }^{30}$

Let us compare the results of Proposition 4 with the empirical observations suggesting more homophily in larger groups. Since we indeed find that, as $n$ increases, the extreme intervals tend to break down into an increasing number of smaller intervals, our result does not necessarily contradict this evidence. Instead, it qualifies it. Proposition 4 highlights the fact that the location within the taste

\footnotetext{
${ }^{30}$ Moreover, in the proof of Proposition 4, we show that any such two agents can disagree on at most one signal for any larger group size (that is, their taste parameters must belong to either a unique interval or to two contiguous intervals of the series $\left\{T_{k}^{n^{\prime}}\right\}_{k=1}^{n^{\prime}-1}$ for any $\left.n^{\prime}>n\right)$.
} 
spectrum may play an important role in identifying these sort of comparative statics: The tendency of larger groups to display more similarity should be stronger for extreme taste parameters than for moderate ones.

The intuition for this result is the following. For simplicity, assume that $q_{\alpha}=q_{\beta} \equiv q$ and that two agents of differing tastes agree on the optimal way to allocate the number of signals $n$. This implies that, for each of these two agents, the marginal utilities of the two signals $\alpha$ and $\beta$ generated by this allocation are roughly the same. Suppose, now, that we give the two agents the possibility to allocate two more signals (that is, $n^{\prime}=n+2$ ). The best way to keep on equalizing the marginal utilities of the signals must be to allocate one signal to source $\alpha$ and the other to $\beta$. Thus, these agents still share the same optimal allocation for $n+2$ signals. Formally, notice that when $k$ of $n$ signals are $\alpha$-signals, the marginal return for an agent of taste $t$ from an $\alpha$-signal is proportional to $t(1-q)^{k}$, while their marginal return from a $\beta$-signal is proportional to $(1-t)(1-q)^{n-k}$. The ratio of these marginal returns remains the same if we increase $k$ by $l$ and $n$ by $2 l$. In other words, if $k \alpha$-signals and $n-k \beta$-signals is an optimal allocation for an agent of taste $t$ when there are $n$ signals available, $k+l$ $\alpha$-signals and $n+l-k \beta$-signals would be an optimal allocation of $n+2 l$ signals for that agent. In particular, the intermediate intervals characterizing stable groups remain the same for even (or odd) group sizes (their number increases with $n$, however). ${ }^{31,32}$

\section{Stability In a Finite Population}

Thus far, our notion of stability has imposed no restrictions on the groups available for the agents to join: Indeed, agents contemplate all possible combinations of tastes when choosing their optimal peer group. This is a good description for very large (strictly speaking, infinite) populations, and allows us to derive a clean characterization of stable groups in different contexts. However, when a finite population of agents is partitioned into groups, there is a restricted set of groups that is conceivably available to an agent. In this section, we study partitions of agents into groups that are "endogenously"

\footnotetext{
${ }^{31}$ For extreme stable groups, the argument is slightly different. Indeed, extreme stable sets contain agents for whom the optimal allocation of $n$ signals is a corner solution, which does not equalize the marginal utility of signals, As the number of signals $n$ increases, more agents whose tastes are not at the extremes of the interval $[0,1]$ tend to reach interior solutions.

${ }^{32}$ Note that the comparative statics with respect to group size $n$ are fully isomorphic to a comparative statics exercise in which the group size is fixed, but we increase the number of signals that each agent acquires from 1 to any $h \geq 1$. In this case, group stability requires all agents in the group to agree on how to allocate $n \times h$ signals. Thus, the same considerations made for changes in group size apply to changes in $h$ as well,
} 
stable. That is, we look for partitions of the population into groups such that no feasible deviation of an agent to a different existing group (or to a singleton), is profitable.

Suppose, then, that there is a finite set of agents $N=\{1, \ldots, l\}$. Let $N_{i} \subseteq N$ be the set of agents with taste $t_{i}$ and let $\left|N_{i}\right|=m_{i}$. Thus, we can write $N=\cup_{i=1}^{r} N_{i}$. Without loss of generality, we assume $t_{1}>\ldots>t_{r}$

In analogy with our baseline setup, the extended game that the agents in the set $N$. play consists of two stages. First, the population $N$ is partitioned into groups. Let $\mathcal{G}=\left\{G_{1}, \ldots, G_{s}\right\}$ be the resulting partition of $N{ }^{33}$ Importantly, here we do not exogenously fix the size of the groups composing the partition $\mathcal{G}$.

The second stage of the game coincides with the information-collection game described in Section 2. In order to extract the effects of finiteness on stability, and not confound them with free rider problems, we focus our discussion on the free-information case. This implies that, within each group $G_{i}$, an information-collection equilibrium entails all agents acquiring a signal and corresponds to the description in Lemma 1. Thus, to complete the analysis of this game, we can focus on the groupformation stage.

We now define stability in this setting as follows.

Definition (Stable Partition) A partition $\mathcal{G}=\left\{G_{1}, . . G_{s}\right\}$ is stable if there exist no $G_{i}, G_{j} \in \mathcal{G}$ and $a \in G_{i}$, such that agent a prefers either the group $G_{j} \cup\{a\}$ or the singleton $\{a\}$ to $G_{i}$.

This notion of a stable partition is reminiscent of the notion of the core, which also requires a type of group stability. Nonetheless, there are several important distinctions. First, the setup is different - cooperative games normally specify group values, rather than individual values within groups that are derived endogenously from a strategic interaction. Second, cooperative solutions (e.g., the core) are more restrictive in that they allow for arbitrary group deviations, not only unilateral ones. Thus, if we take a group's value to be the sum of its members' expected utilities, the set of stable groups we look at corresponds to a superset of the core, which in this setting corresponds to the grand coalition onily. Finally, the core identifies stable allocations of resources, rather than a characterization of the ${ }^{33}$ So that $G_{i} \cap G_{j}=\varnothing$ for all $i \neq j$ and $\bigcup_{i=1}^{s} G_{i}=N$. 
emerging partitions themselves.

Since adding a member to a group is costless, and a new group member provides more information. to the other members in the information-collection stage, groups always benefit from adding more members. Thus, we do not have to attend to any issues pertaining to the willingness of a group to accept a new member. ${ }^{34}$

Certainly, the grand coalition $\mathcal{G}=\{N\}$ is always a stable partition and, in fact, it is the welfaremaximizing partition. ${ }^{35}$ In what follows, we explore whether inefficient solutions can arise, i.e., we study the stability of other partitions.

As a first step, Lemma 3 addresses two properties of any stable partition, namely, agents of similar. tastes cluster together, and all agents sharing the same taste must be contained in the same group.

Lemma 3 (Consecutive and Minimal Groups) In any stable partition $\mathcal{G}$,

1. All groups are consecutive, i.e., if $t_{i}>t_{j}>t_{h}$; and two agents with tastes $t_{i}$ and $t_{h}$ are in a group $G \in \mathcal{G}$ then any agent. with taste $t_{j}$ must be in $G$ as well;

2. All agents with the same taste are contained in the same group, i.e., for each $t_{i}$, there exists a unique $G \in \mathcal{G}$ such that $N_{i} \subseteq G$.

Point (1) of Lemma 3 is a sorting result in the spirit of the analysis of point (1). in Proposition 2: In any stable partition groups are characterized by individuals that are similar enough in taste. ${ }^{36}$ Point (2) of Lemma 3 implies that the maximal number of groups contained in a stable partition is bounded by the total number $r$ of different taste parameters in the population.

Intuitively, consider the first part of the lemma. If the agent of type $t_{j}$ prefers a different group $G^{\prime}$ than the group $G$ containing agents of types $t_{i}$ and $t_{h}$, then $G$ must involve the collection of either more $\alpha$-signals or more $\beta$-signals relative to $G^{\prime}$ (or else the $t_{j}$-type agent would benefit by switching to $G$ ). Suppose more $\alpha$-signals are collected in $G$. Since the agent of type $t_{h}$ care even more than

\footnotetext{
${ }^{34}$ If communication from agent to agent is costly, a group may be unwilling to accept new members, or prefer particular: new members to others. We elaborate on this in Section 6.

${ }^{35}$ This is in contrast to the underlying assumption in Demange (1994), who studies a similar question in a setup where the grand coalition generates inefficient outcomes and, unlike here, any collection of agents can jointly deviate.

${ }^{36}$ The notion of 'consecutive groups' is reminiscent of the one adopted by Greenberg and Weber (1986) in the context of characterizing the core in Tiebout multi-jurisdictional economies.
} 
the $t_{j}$-type agent about issue $A$ and her switch to $G^{\prime}$ would assure an even greater number of signals collected (in $G^{\prime}$ augmented with her participation), the $t_{h}$-type.agent cannot be optimizing.

The intuition for point (2) of Lemma 3 is similar. Suppose that two agents $a$ and $a^{\prime}$ with the same taste parameter $t_{i}$ belong to two different groups $G$ and $G^{\prime}$, respectively. Consider the agent $a$ of taste parameter $t_{i}$ in $G$. Since $\mathcal{G}$ is stable, this agent must prefer to stay in $G$ rather than being in $G^{\prime} \cup\{a\}$. However, since $a$ and $a^{\prime}$ have the same tastes, and a shift of agent $a^{\prime}$ to group $G$ would entail even more information collected, this implies that agent $a^{\prime}$ must prefer being in $G \cup\left\{a^{\prime}\right\}$ rather than in $G^{\prime}$, which contradicts the stability of $\mathcal{G}$.

Next, we define the fully segregated partition to be the partition in which each set is formed only by agents of the same type, the partition $\mathcal{G}=\left\{N_{1}, N_{2}, \ldots, N_{r}\right\}$.

Since Lemma 3 guarantees that agents of the same type cannot be divided across different groups in any stable partition, we can conclude that the fully segregated partition is the least efficient partition that could be stable. In what follows, we aim to identify the conditions under which this partition is, indeed, stable.

Consider a fully segregated partition, and consider an agent $a$ of type $t_{i}$ belonging to group $N_{i}$ who is considering deviating to the group $N_{j} \cup\{a\}$. By staying in $N_{i}$, agent $a$ is able to implement her optimal allocation of $m_{i}$ signals across the sources $\alpha$ and $\beta$ in the information-collection stage. Deviating to $N_{j} \cup\{a\}$ cannot be strictly profitable if $m_{j}<m_{i}$. On the other hand, if $m_{j} \geq m_{i}$, the group $N_{j} \cup\{a\}$ is bigger than $N_{i}$, which implies more information gathered in the informationcollection stage. Thus, a deviation to $N_{j} \cup\{a\}$ tends to be less beneficial if: (i) the taste parameters $t_{i}$ and $t_{j}$ are far from one another (as agent $a$ finds herself in a group in which other agents' optimal choice of signal sources is very different than hers); and (ii) the size of the groups $m_{i}$ and $m_{j}$ are close to one another (as agent $a$ benefits less from an increase in group size). To focus on the first issue (identifying taste distributions that allow for segregation) and simplify our exposition, from now on we assume that $\left|N_{i}\right|=m$ for all $i$ (we discuss the implications of relaxing this assumption at the end of this section). A consequence of the above is that a necessary condition for full segregation to be a stable partition is as follows. 
Lemma 4 (Heterogeneity and Segregation) Full segregation is a stable partition only if there are no $t_{i} \neq t_{j}$ sharing the same optimal allocation of $m$ signals, $\underline{i}_{\text {. }}$, , there are no $t_{i} \neq t_{j}$ such that $n^{\alpha}\left(t_{i}\right)=n^{\alpha}\left(t_{j}\right)$.

The complete characterization of the necessary and sufficient conditions for the fully segregated partition to be stable directly exploits the characterization of the stable groups via the sequence $\left\{T_{k}^{n}\right\}$ developed in Proposition 2. In order to ease the description of our results, we assume that the sufficient conditions for the full sequence $\left\{T_{k}^{m}\right\}_{k=0}^{m}$ to follow the pattern described in point (2) of Proposition 2 are met. ${ }^{37}$

Consider an agent $a$. with taste parameter $t_{i} \in\left\{t_{1}, \ldots, t_{r}\right\}$. In the fully segregated partition, such an agent belongs to the group $N_{i}$. To show stability, we need to consider all possible deviations of agent $a$ to any set $N_{j} \cup\{a\}, j \neq i$. Since $\left|N_{i}\right|=m$ for all $i=1, . ., r$, checking that deviations to the groups closest in tastes, $N_{i-1} \cup\{a\}$ and $N_{i+1} \cup\{a\}$, are not profitable is sufficient. ${ }^{38}$. Thus, requiring a deviation from $N_{i}$ to $N_{i+1} \cup\{a\}$ not to be profitable allows us to identify an upper bound for $t_{i+1}$ for segregation to be stable. Similarly, requiring a deviation from $N_{i}$ to $N_{i-1} \cup\{a\}$ not to be profitable allows us to identify a lower bound for $t_{i-1}$ for segregation to be stable. Such bounds can be used to define an interval $\mathcal{T}_{i}$ around each $t_{i}$ such that full segregation is stable if and only if for any $i \neq j$, $t_{j} \notin \mathcal{T}_{i}$. That is, $t_{i}$ and $t_{j}$ are sufficiently far from each other.

The idea that agents in other groups have sufficiently different tastes is then captured by them agreeing (among their cohesive groups of $m$ agents) on very different allocations of signals. In particular, it can be shown that the intervals $\mathcal{T}_{i}$ correspond to unions of the original intervals in the sequence $\left\{T_{k}^{m}\right\}_{k=0}^{m}$. Figure 4 illustrates graphically the structure of the intervals $\mathcal{T}_{i}$ and $\mathcal{T}_{i+1}$ as unions of sets of the original sequence $\left\{T_{k}^{m}\right\}_{k=0}^{m}$.

We now turn to the robustness of the basic comparative statics obtained in Section 3. Recall that the main insight from our baseline analysis was that similarity in stable groups is stronger for extreme than for moderate tastes. In the same spirit, in the finite population case we show that segregation is easier to achieve for extreme types than for moderate ones. Intuitively, suppose that agent $a$ has

\footnotetext{
${ }^{37}$ These conditions are briefly discussed in Footnote 19 and formally derived in the Appendix. Recall that they amount to $q_{\alpha}, q_{\beta}$, and $m$ being high enough.

${ }^{38}$ We have to consider just one potential deviation for agents with extreme tastes $t_{1}$ and $t_{r}$.
} 


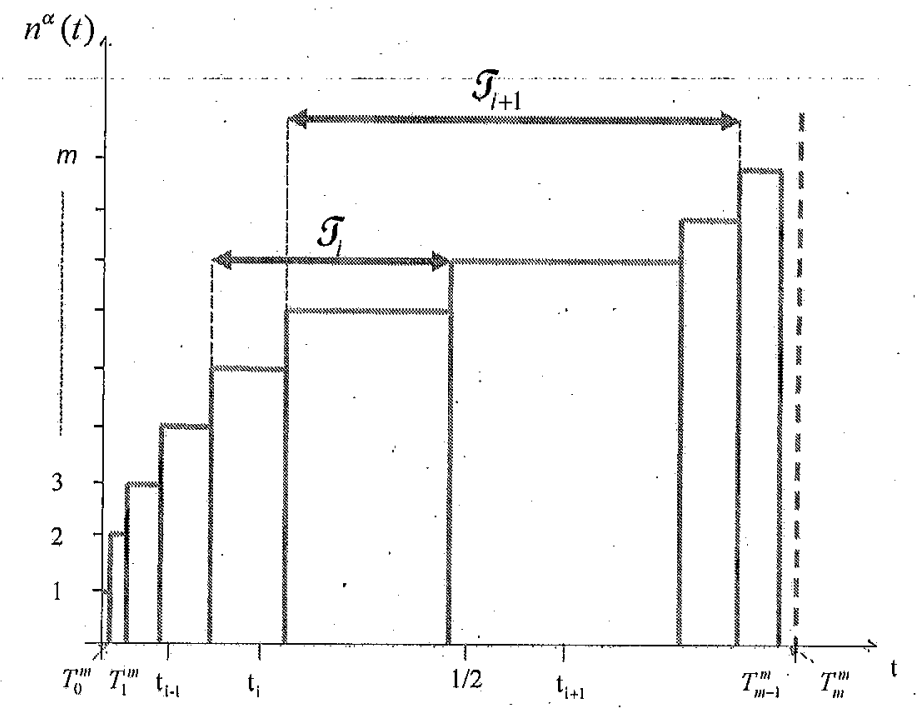

Figure 4: Segregation Intervals

taste parameter $t_{i}$, and consider a deviation from the group $N_{i}$ to $N_{i+1} \cup\{a\}$. As mentioned above, the benefit for agent a from joining $N_{i+1}$ is to be in a group formed by $m+1$ agents rather than $m$ agents. On the other hand, the cost of joining $N_{i+1}$ comes from the expected sub-optimal allocation. of $m+1$ signals across the sources $\alpha$ and $\beta$. From Proposition 2, we know that the intervals $\left\{T_{k}^{m}\right\}_{k=0}^{m}$, each containing all the t's that correspond to the same optimal allocation of $m$ signals, are narrower for extreme taste parameters and wider for moderate taste parameters. Thus, for a given distance between $t_{i}$ and $t_{i+1}$, the disagreement between agent $a$ and the agents in $N_{i+1}$ is stronger if $t_{i}$ and $t_{i+1}$ are taste parameters closer to the extremes than if they are moderate. In other words, the intervals $\left\{\mathcal{T}_{i}\right\}$ follow a similar pattern described in Section 3 for the interval sequence $\left\{T_{k}^{m}\right\}_{k=0}^{m}$.

This implies that moderate individuals can be quite heterogeneous without segregation emerging, whereas extremists can be less heterogeneous in comparison and still allow for multiple small and homogenous groups. In particular, in the case of a finite population uniformly distributed on equidistant points on the interval $[0,1]$, the most segregated stable partition that can emerge will tend to display large, heterogeneous groups of moderate agents and multiple, smaller groups of extremists.

Proposition 5 formalizes the above discussion. 
Proposition 5 (Segregation) Any $t_{i}$ is contained in an interval $\mathcal{T}_{i}$ such that:

1. Full segregation is a stable partition if and only if for any $i \neq j, t_{i}$ and $t_{j}$ are sufficiently far from each other: $t_{j} \notin \mathcal{T}_{i}$.

2. Full segregation is easier to sustain as stable for extreme tastes than for moderate tastes: there exists $i$ such that the intervals $\mathcal{T}_{1}, \mathcal{T}_{2}, \ldots, \mathcal{T}_{i}$ are increasing in length; similarly, there exists $j$ such that the intervals $\mathcal{T}_{j}, \mathcal{T}_{j+1}, \ldots, \mathcal{T}_{s}$ are decreasing in length.

To conclude this section, let us address the implications of relaxing the assumption that $\left|N_{i}\right|=m$ for all $i$, and allowing for the sets of agents sharing the same taste parameter to be of different sizes. Note that agents of the same taste will always want to be in the same group, following the lines of Lemma 3 above. Now, the larger the number of individuals of a particular taste parameter is, the more appealing the group that contains these individuals becomes (by the sheer volume of information they collect). Therefore, in order to achieve stable segregation, the other agents need to be further away in terms of their preferences. So, the band around a particular type that guarantees segregation increases in width with the number of agents of that type.

This result can be applied to derive straightforward comparative statics with respect to the population distribution. For example, consider two cumulative distributions over tastes in the population $G$ and $G^{\prime}$ such that $G$ is a mean-preserving spread of $G^{\prime}$. In this case, the most segregated partition corresponding to $G^{\prime}$ will be characterized by larger and more heterogeneous groups of moderates, and smaller and more fractioned groups of extremists relative to the most segregated partition corresponding to $G$,

\section{Concluston and EXTEnsions}

The model developed in this paper addresses the properties of peer groups that arise in equilibrium when individuals have different tastes and share the information they gather with members of their group. If information gathering costs are low, stable groups are composed of individuals that are sufficiently similar in tastes. This similarity is more pronounced for extrome agents than for moderate ones. If information gathering costs are high, stable groups display taste polarization, which becomes more extreme as the group size increases. Finally; when the population is small, it is natural to 
consider stable partitions of the population into groups such that agents'. deviations are restricted to joining one of the other groups in the population, or creating a group by themselves. In this setup, we showed that full segregation is easier to achieve for agents with extreme tastes than for agents with moderate ones. In what follows, we discuss some natural extensions of our model.

6.1. General Production Functions. A crucial element of our analysis is that agents' utilities exhibit decreasing marginal returns with respect to the total number of signals collected from each source. The more signals an agent receives (directly or indirectly) on a particular issue, the lower the value of an additional signal on that issue. Our information-based setup pinned down precisely the shape of agents' utilities corresponding to any allocation of signals collected within a group. Nonetheless, the idea that utilities exhibit decreasing marginal returns to effort is a recurring theme in many models, where effort can have many meanings, ranging from information collection, as in the current paper, to investment levels in projects, to physical exertion on the job, etc.

The main assumption in our model is the way units of effort on each dimension (in our case, signals on different issues) are transformed into utiles (throughout the paper, captured by (1)). In a more 'general setup, agents have to invest effort on two dimensions $\alpha$ and $\beta$ that correspond to two different public projects (e.g., volunteering, freeware development, student associations activities, and many more). Each agent can invest a unit of effort on either dimension (for instance, signals as in our baseline model). To compare this setting with the baseline results in Section 3.2.1, assume that effort comes at no cost (or sufficiently low costs). Agents differ in how much they care about either project, and utility takes the form $U\left(t, k^{\alpha}, k^{\beta}\right)$, where $t \in[0,1]$ is the agent's type, and $k^{x}$ is the number of units of effort invested in dimension $x, x=\alpha, \beta$. We assume that $U$ is concave in $k^{\alpha}$ and $k^{\beta}$.

For an agent of taste $t$, the optimal number of $\alpha$ units out of $n$ is given by $n^{\alpha}(t)=\left\lfloor m^{\alpha}(t)\right\rfloor$, where $m^{\alpha}(t)$ solves

$$
U\left(t, m^{\alpha}(t), n-m^{\alpha}(t)\right)=U\left(t, m^{\alpha}(t)-1, n-m^{\alpha}(t)+1\right)
$$

Note that the techniques introduced throughout the paper to characterize group stability can still be used.

First, let us consider the sorting result in point (1) of Proposition 2. For sorting to arise, the set 
of types that agree on a particular allocation of units of effort $\left(k^{\alpha} ; k^{\beta}\right)$ must be a connected set. In particular,- if the optimal-allocation-reacts-monotonically-to the-type-t-sorting emerges:- Assuming$U$ is well-behaved, monotonic behavior of the optimal allocation corresponds to $\frac{\partial^{2} U}{\partial t \partial k^{\alpha}}$ being signed, which is, up to a re-directioning of the type space, essentially a single crossing property.

Let us now turn to the comparative statics pertaining to the potential dispersion within stable groups, i.e., point (2) of Proposition 2. Naturally, the generalization of this result depends on the shape of $U$. In order to make the comparison with the basic model of the paper transparent, suppose $U$ is linear in t. Agents' utilities can then be described using two production functions, $f_{\alpha}\left(k^{\alpha}\right)$ and $f_{\beta}\left(k^{\beta}\right)$ that correspond to the mappings between effort units and utility returns on dimensions $\alpha$ and $\beta$, respectively. We assume that $f_{\alpha}$ and $f_{\beta}$ are increasing and concave. ${ }^{39}$ Thus, if $k^{x}$ is the number of units of effort invested in the project $f_{x}, x=\alpha, \beta$, the general utility for an agent of taste $t \in[0,1]$ is given by:

$$
\tilde{U}\left(t, k^{\alpha}, k^{\beta}\right) \equiv t f_{\alpha}\left(k^{\alpha}\right)+(1-t) f_{\beta}\left(k^{\beta}\right)
$$

which generalizes (1). ${ }^{40}$

For $x=\alpha, \beta$, define $\phi_{x}(k) \equiv f_{x}(k+1)-f_{x}(k)$ and $\phi_{x}^{(1)}(k)=f_{x}^{\prime}(k+1)-f_{x}^{\prime}(k)$. Applying the Implicit Function Theorem to condition (6), we get

$$
\frac{d m^{\alpha}(t)}{d t}=\frac{-\frac{1}{t(1-t)}}{\frac{\phi_{\alpha}^{(1)}\left(m^{\alpha}-1\right)}{\phi_{\alpha}\left(m^{\alpha}-1\right)}+\frac{\phi_{\beta}^{(1)}\left(n-m^{\alpha}\right)}{\phi_{\beta}\left(n-m^{\alpha}\right)}},
$$

which is positive. ${ }^{41}$ Recall that, in our previous setting, the sign of $\frac{d^{2} m^{\alpha}(t)}{d t^{2}}$ is positive for $t>\frac{1}{2}$ and negative for $t<\frac{1}{2}$, which determines the interval pattern described in point (2) of Proposition 2. In this more general case, differentiating again, and rearranging terms, it is easy to show that the sign of

\footnotetext{
${ }^{39}$ Note that in terms of our information story, this allows for signals within a dimension to be correlated.

${ }^{40}$ In our analysis in the previous sections, $f_{\alpha}\left(k^{\alpha}\right)=1-\frac{1}{2}\left(1-q_{\alpha}\right)^{k^{\alpha}}$ and $f_{\beta}\left(k^{\beta}\right)=1-\frac{1}{2}\left(1-q_{\beta}\right)^{k^{\beta}}$.

${ }^{41}$ Our assumptions on the production functions guarantee that $\phi_{x}(k)>0$ while $\phi_{x}^{(1)}(k)<0$ for $x=\alpha, \beta$.
} 
$\frac{d^{2} m^{\alpha}(t)}{d t^{2}}$ depends on the third derivative of the production functions. ${ }^{42}$ In general, the third derivative of the production functions plays an important role in affecting the curvature of $m^{\alpha}$ and, consequently, the relative lengths of the intervals that characterize the stable groups. Intuitively, as described in Section 3.2.1, the curvature of $m^{\alpha}(t)$ is the result of two effects: as $t$ increases, the marginal utility of a unit of effort on dimension $\alpha$ increases. On the other hand, since the optimal number of these contributions increases with $t$, the marginal returns of any effort on dimension $\alpha$ decreases with $t$. For $m^{\alpha}(t)$ to be convex to the right of $t=\frac{1}{2}$ (and, analogously, concave to the left of $t=\frac{1}{2}$ ), the first force must prevail. In particular, the marginal return of $f_{\alpha}$ cannot decrease too rapidly. This, in turn, translates into bounds on the magnitudes of the third derivative of the production functions.

To shed some light on the link between the production functions and the structure of the stable groups, assume that $f \equiv f_{\alpha}=f_{\beta}$. It is easy to show that

$$
\operatorname{sign}\left[f^{(l)}(k)\right]=(-1)^{l+1} \quad \text { for } l \leqslant 3
$$

is a sufficient condition for the function $m^{\alpha}(t)$ to be convex for sufficiently large $t$ and concave for sufficiently low $t$. Thus, in those ranges of $t$, the characterization of Section 3.2.1 still holds. That is, stability requires more similarity for more-extreme tastes.

Two examples of commonly used production functions that satisfy condition (7) are the following:

1. $f(k)=k^{\gamma}$, where $\gamma \in(0,1)$. Note that

$$
f^{(l)}(k)=\left[\prod_{j=0}^{l-1}(\gamma-j)\right] k^{\gamma-l} \quad \text { for all } l>0
$$

which satisfies (7).

\footnotetext{
${ }^{42}$ Formally, for $x=\alpha, \beta$, denoting $\phi_{x}^{(2)}(k)=f_{m}^{\prime \prime}(k+1)-f_{x}^{\prime \prime}(k), \operatorname{sign}\left(\frac{d^{2} m^{\alpha}(t)}{d t^{2}}\right)$ coincides with the sign of the following: $\frac{d m^{\alpha}(t)}{d t}\left[\phi_{\alpha}^{(1)}\left(m^{\alpha}-1\right)-\phi_{\beta}^{(1)}\left(n-m^{\alpha}\right)\right] \times$$$
\left\{\frac{d m^{\alpha}(t)}{d t}\left[t \phi_{\alpha}^{(1)}\left(m^{\alpha}-1\right)+(1-t) \phi_{\beta}^{(1)}\left(n-m^{\alpha}\right)\right]-\left[\phi_{\alpha}\left(m^{\alpha}-1\right)+\phi_{\beta}\left(n-m^{\alpha}\right)\right]\right\}+
$$$$
\frac{d m^{\alpha}(t)}{d t}\left[t \phi_{\alpha}^{(2)}\left(m^{\alpha}-1\right)-(1-t) \phi_{\beta}^{(2)}\left(n-m^{\alpha}\right)\right] \times\left[\phi_{\alpha}\left(m^{\alpha}-1\right)+\phi_{\beta}\left(n-m^{\alpha}\right)\right] .
$$ 
2. $f(k)=1-e^{-k \delta}$, where $\delta>0$. Here,

$$
f^{(\bar{l})}(k)=(-1)^{l+1} \delta^{l} e^{-k \delta} \quad \text { for all } l>0
$$

so that, again, (7) is satisfied.

Finally, in the same spirit of Proposition 4, it is possible to show that, under conditions that bound $\frac{d m^{\alpha}(t)}{d t}$ above uniformly, the subintervals of types that agree on the optimal allocation of $n$ signals across the two dimensions do not converge to singletons as the group 'size diverges, ${ }^{43}$

6.2. Multiple Issues. Our results extend to a multi-dimensional setting with $h>2$ issues. In this case, the type space is $T=\left\{\left(t^{1}, . ., t^{h}\right) \geq 0 \mid \sum_{l=1}^{h} t^{l}=1\right\}$. Each $t^{l}$ represents the weight an individual assigns to issue $l=1, . ., h$, and the accuracy of a signal on issue $l$ is given by $q_{l}$.

In this setting, the analysis is essentially analogous to that presented for two dimensions in Section 3.2. If information gathering is free, as in Section 3.2.1, stable groups are composed of agents who agree on an allocation of signals $\left(k_{1}, \ldots, k_{h}\right)$ across the $h$ issues. Stable groups are then characterized by $(h-1)$-dimensional polyhedra, which display patterns similar to those described in point (2) of Proposition 2.

For illustration purposes, consider the simple case of 3 dimensions and identical accuracies of signals across the dimensions given by $q$. To identify the sets of $\left(t_{1}, t_{2}, t_{3}\right)$ that agree on an allocation $\left(k_{1}, k_{2}, k_{3}\right)$ of signals across the three dimensions, one has to look at 6 relevant constraints, corresponding to a shift of one signal from one dimension to the other. For example, an agent would like the allocation $\left(k_{1}, k_{2}, \dot{k}_{3}\right)$ over $\left(k_{1}-1, k_{2}+1, k_{3}\right)$ whenever

$$
t_{1}(1-q)^{k_{1}-1} q \geq t_{2}(1-q)^{k_{2}} q \Longleftrightarrow t_{1} \geq(1-q)^{k_{2}-k_{1}+1} t_{2}
$$

Similar inequalities correspond to the other 5 signal shifts.

Three aspects are important to note: (i) The sorting result in point (1) of Proposition 2 (i.e., the sets of taste parameters sharing the same optimal allocation of signals being convex) holds in this setting. As discussed in Section 6.1 for the two-dimensional case, it does not depend crucially on

\footnotetext{
${ }^{d 3}$ Namely, we need to require that for any $t \in(0,1)$ there exists $v$ such that for all $n, \frac{d m^{\alpha}(t)}{d t}<v$.
} 
the linear specification of the preference or on the specific production functions we have adopted; (ii)

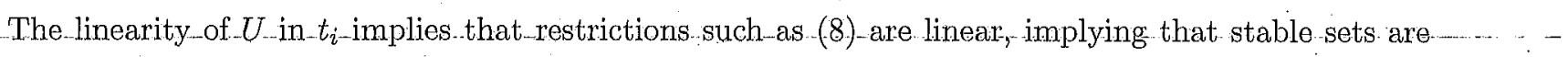
identified by polyhedra; (iii). Finally, it is straightforward to see that the set allowing for the maximal heterogeneity between types (i.e., the largest maximal Euclidean distance between two types) contains types that are moderate - that is, those that agree on an equal allocation of signals across the multiple dimensions; This comparative statics mirrors point (2) of Proposition 2.

The analysis corresponding to the costly information case in Section 3.2.2 can also be directly extended to the multi-dimensional setting. Indeed, agents that are extremist on one particular dimension are still the ones with the highest incentives to collect information on that dimension. Thus, for any dimension $l=1, . ., h$, we can identify the maximal attainable number of signals that can be collected in equilibrium as $n_{\max }^{l}$ : Whenever $n \geq \sum_{l=1}^{h} n_{\max }^{l}$, extremely polarized groups (i.e., groups containing extremists on each dimension) are stable, as in Proposition 3A: On the other hand, if the group size is small enough, stability entails the acquisition of a signal profile no different than that acquired in the free-information case, and the characterization of stable groups is similar to that in Proposition 3B.

6.3. Adding New Members. In the setup presented in Sections 2, 3, and 4 the group size is fixed. This assumption is relaxed in Section 5, where individuals can opt to leave their group and join another one. However, in that analysis, the other group members always benefit (at least weakly) from the addition of a member of any taste. Nonetheless, if adding new members is limited or costly, a group could prefer certain additional members over others, or stop accepting new members altogether. As a natural extension, then, we now consider the problem of a group that is given the opportunity to add one new member and has to select the member's taste parameter.

Consider a group of agents with tastes $t_{1}>\ldots>t_{n}$ and let $k^{*}$ be the number of $\alpha$-signals collected in equilibrium in this group. Suppose that an agent of taste $t^{P}$ (the "principal") chooses an additional agent for the group. ${ }^{44}$

The following proposition illustrates the optimal range of tastes for an additional member that an

\footnotetext{
${ }^{44}$ The principal can be thought of as contemplating the adclition of an expert to an already existing team, a new member to a club, new peers to a circle of friends, etc. Alternatively, the principal can be thought of as the pivotal member in a group using an election to determine the tastes of an additional member in any of the above contexts. If adding a new member requires unanimity, the principal is one of the agents with most extreme tastes. In general, the principal may or may not be part of the original group of agents.
} 
agent with taste parameter $t^{P}$ would choose.

\section{Proposition 6 (Optimal Additional Members) There exists $\widetilde{t} \in[0,1]$ such that}

1. If $t_{k^{*}+1}<\tilde{t}<t^{P}$, then any agent with taste $t \geq \tilde{t}$ is optimal.

2. If $t^{P}<\widetilde{t}<t_{k^{*}+1}$, then any agent with taste $t<\widetilde{t}$ is optimal.

3. In all other cases, any agent is optimal.

The proof is straightforward, and, therefore, omitted. Intuitively, if, in the original group, precisely $k^{*}$ agents chose source $\alpha$, then adding a member can shift the number of agents choosing source $\alpha$ to $\hat{k} \in\left\{k^{*}, k^{*}+1\right\}$. Thus, when the principal cares sufficiently about the realization of issue $A$, she would like $k^{*}+1 \alpha$-signals to be collected in the new group. Then, two cases are possible: if there are already $k_{-}^{*}+1$ agents who are willing to choose $\alpha$-signals in a group of $n+1$ agents (i.e.; they agree with the principal on the allocation of the new available signal), then the additional member's tastes are inconsequential. Otherwise, a new member who agrees with the principal on the allocation of the new available signal has to be introduced. ${ }^{45}$

6.4. Externalities. In our setup, once information is collected, all agents in the group agree on which is the more likely realization of each issue. Therefore, adding positive action externalities (say, by requiring all agents in a group to make the same collective decision, determined by a threshold vote), would not alter our results. A natural extension of our setup would allow for non-trivial externalities in actions. In our model, this would require adding another dimension of heterogeneity, one pertaining to reactions to information. That is, agents may differ in their inclinations to choose the action 1 on either issue. ${ }^{46}$ Externalities in actions would then take the form of agents caring positively about the number of agents in their group who make similar decisions to theirs. ${ }^{47}$ In such a model, there is an interplay between 'both types of heterogeneity (that regarding issue weights, and that regarding actions

\footnotetext{
${ }^{45}$ It follows that $\tilde{t}$ is the taste parameter that identifies an additional agent who would be indifferent between $k^{*}$ and $k^{*}+1 \alpha$-signals (in the new group of $n+1$ agents).

${ }^{46}$ For our information structure, as long as each agent wants to match the realization of each issue to some extent, this type of heterogeneity would play a role only when the realization of one issue does not get revealed in the information collection stage.

${ }^{47}$ For example, if $A$ stands for food and $B$ stands for books, the two realizations within each issue may be thought of as two restaurants and two bookclubs, respectively, and agents care about how many of their peers join them in each.
} 
within each issue). Indeed, action externalities may affect agents' incentives to acquire information on .oither_dimension. 48 In_our baseline_analysis,_such _a_setup_would,_if_anything;,_generate_stronger similarity with respect to issue weights. It would also lead to limited heterogeneity in tastes over choices within each issue.

In realty, there are many examples in which there are no action externalities (e.g., Internet forums, long-distance friendships, online social networks, etc.). Conceptually, one of the contributions of our analysis is to show that, even in the absence of action externalities, homophily can still arise through pure information-based motives. Moreover, information-based homophily follows some interesting comparative statics patterns.

\section{APPENDIX}

Proof of Lemma 1. Let $t_{1} \geq \ldots \geq t_{n}$. Each agent has to decide whether to acquire an $\alpha$-signal, a $\beta$-signal, or forgo information gathering.

To construct an efficient equilibrium in the information-collection game, let $\mu^{\alpha}$ be the maximal integer $h$ such that $\frac{t_{h}}{2}\left(1-q^{\alpha}\right)^{h-1} q^{\alpha} \geq c$ (this is inequality (3) in the text). Similarly, let $\mu^{\beta}$ be the minimal integer $h$ such that $\frac{\left(1-t_{h}\right)}{2}\left(1-q^{\beta}\right)^{h-1} q^{\beta} \geq c$ (this is inequality (4) in the text)..

First, consider the case in which $\mu^{\alpha}+1 \geq \mu^{\beta}$, so that all agents could be induced to acquire information. We first construct an equilibrium entailing all agents acquiring information. We consider an equilibrium as proposed by the Lemma's claim, so that $\tau^{\alpha}=\tau^{\beta}-1 \equiv \tau^{*}$. Note that if an agent of taste $t$ prefers getting an $\alpha$-signal over a $\beta$-signal, so would any agent of taste $t^{\prime}>t$. Similarly, if an agent of taste $t$ prefers getting a $\beta$-signal, so would any agent of taste $t^{\prime}<t$. In such an equilibrium, the agent with the lowest taste parameter who chooses an $\alpha$-signal is the agent with taste $t_{\tau^{*}}$. From our tie-breaking rule, it follows that the threshold $\tau^{*}$ is determined as the maximal $\tau \in\{1, \ldots, n\}$ for which agent $\tau$ weakly prefers an $\alpha$-signal over a $\beta$-signal, or for which

$$
U\left(t_{\tau}, \tau, n-\tau\right) \geq U\left(t_{\tau}, \tau-1, n-\tau+1\right)
$$

is satisfied, This inequality is constraint (2) for taste $t_{\tau}$. If (2) is not satisfied for any agent in the

\footnotetext{
${ }^{48}$ Note that in such a setup, an agent needs to worry about the effect of an additional signal on either issue not only on their own action, but also on others'.
} 
group (i.e., $\left.U\left(t_{1}, 0, n\right)>U\left(t_{1}, 1, n-1\right)\right)$, then $\tau^{\alpha}=\tau^{*}=0$ and $\tau^{\beta}=1$ defines an equilibrium. In order to show that choosing $\tau^{*}=0$ if (2) is not satisfied for any positive integer and $\tau^{*}$ as the maximal integer between 1 and $n$ satisfying (2) otherwise defines an equilibrium all that remains to be shown is that incentives to acquire information are satisfied. Notice that for any agent $\tau \leq \tau^{*}$,

$$
\begin{aligned}
U\left(t_{\tau}, \tau, n-\tau\right)-U\left(t_{\tau}, \tau-1, n-\tau+1\right) & =\left[U\left(t_{\tau}, \tau, n-\tau\right)-U\left(t_{\tau}, \tau-1, n-\tau\right)\right] \\
& -\left[U\left(t_{\tau}, \tau-1, n-\tau+1\right)-U\left(t_{\tau}, \tau-1, n-\tau\right)\right] \geq 0
\end{aligned}
$$

and so, the incentives to acquire an $\alpha$-signal are greater than those to acquire a $\beta$-signal. Similarly, for agents $\tau>\tau^{*}$, the incentives to acquire a $\beta$-signal ${ }^{\star}$ are greater than those to acquire an $\alpha$-signal. Since $\mu^{\alpha}+1 \geq \mu^{\beta}$, it follows that the identified profile constitutes an equilibrium.

Consider now the case in which $\mu^{\alpha}+1 .<\mu^{\beta}$, and define $\tau^{\alpha} \equiv \mu^{\alpha}$ and $\tau^{\beta} \equiv \mu^{\beta}$. From our definitions of $\mu^{\alpha}$ and $\mu^{\beta}$, in order to illustrate that the suggested profile constitutes an equilibrium, all that remains to be shown is that an agent acquiring a signal $x=\alpha, \beta$ does not prefer to acquire a signal $y \neq x$ when all other agents follow the profile. Indeed, suppose that $i \leq \tau^{\alpha}<\tau^{\beta}$ and observe that

$$
U\left(t_{i}, \tau^{\alpha}, n-\tau^{\beta}+1\right)-c \geq U\left(t_{i}, \tau^{\alpha}-1, n-\tau^{\beta}+1\right)>U\left(t_{i}, \tau^{\alpha}-1, n-\tau^{\beta}+2\right)-c,
$$

where the first inequality follows from inequality (3), and the second from the fact that $\mu^{\alpha}+1<\mu^{\beta}$. Thus, an agent of taste $t_{i}$ does not profit from deviating to a choice of a $\beta$-signal instead of an $\alpha$-signal. An analogous argument holds for $i \geq \mu^{\beta}>\mu^{\alpha}$.

Suppose now that there are two equilibria, one of which entails $k^{\alpha} \alpha$-signal and $k^{\beta} \beta$-signals and one that entails $\tilde{k}^{\alpha} \alpha$-signals and $\tilde{k}^{\beta} \beta$-signals. We now show that either $k^{\alpha} \leq \tilde{k}^{\alpha}$ and $k^{\beta} \leq \tilde{k}^{\beta}$ or $k^{\alpha} \geq \tilde{k}^{\alpha}$ and $k^{\beta} \geq \tilde{k}^{\beta}$. Suppose, for instance, that $k^{\alpha}>\tilde{k}^{\alpha}$ and $k^{\beta}<\tilde{k}^{\beta}$. This implies that there is an agent with taste $t_{i}$ that in the first equilibrium acquires an $\alpha$-signal, and in the second equilibrium acquires either no signal or a $\beta$-signal. However, notice that for any such $t_{i}$

$$
\begin{gathered}
U\left(t_{i}, k^{\alpha}, k^{\beta}\right)-U\left(t_{i}, k^{\alpha}-1, k^{\beta}\right) \leq U\left(t_{i}, \tilde{k}^{\alpha}+1, \tilde{k}^{\beta}\right)-U\left(t_{i}, \tilde{k}^{\alpha}, \tilde{k}^{\beta}\right) \text { and } \\
U\left(t_{i}, k^{\alpha}, k^{\beta}\right)-U\left(t_{i}, k^{\alpha}-1, k^{\beta}+1\right) \leq U\left(t_{i}, \tilde{k}^{\alpha}+1, \tilde{k}^{\beta}-1\right)-U\left(t_{i}, \tilde{k}^{\alpha}, \tilde{k}^{\beta}\right),
\end{gathered}
$$

in contradiction to $t_{i}$ using a best response in both equilibria: Other cases are shown similarly. 
Since the equilibrium identified above establishes the maximal volume of signals, it follows that it _is_also_the_most_efficient._Furthermore,_whenever_c_is_small_enough_so_that_any_equilibrium_entails_all agents acquiring information, the equilibrium number of $\alpha$-signals is determined uniquely.

Proof of Proposition 1. In order to show that the conditions in (1) are sufficient, we construct an optimal group as follows. If an agent is selected to be part of the group and is to collect, say, an $\alpha$-signal, then she must have a taste parameter $t^{\prime}$ such that (i) she prefers to gather an $\alpha$-signal rather than a $\beta$-signal, that is, $t^{\prime} \geq l(t)$, as described in the text for the $c=0$ case, and (ii) she has enough incentives to gather an $\alpha$-signal rather than no signal, that is $n_{c}^{x}\left(t^{\prime}\right) \geq n^{x}(t)$. Thus, $n^{x}(t) \leq n_{\max }^{x}$ is a necessary condition for the unconstrained optimal allocation $\left(n^{\alpha}(t), n^{\beta}(t)\right)$ to be achievable. Moreover, to achieve her optimal allocation $\left(n^{\alpha}(t), n^{\beta}(t)\right)$, the agent of taste parameter $t$ has to have incentives herself to acquire a signal from at least one source. That is, $n^{x}(t) \leq n_{c}^{x}(t)$ for at least one $x \in\{\alpha, \beta\}$. Note that if such incentives cannot be provided, meaning $n^{x}(t)>n_{c}^{x}(t)$ for $x=\alpha, \beta$, an optimal group would entail the optimal allocation short of one signal.

To show (2), suppose, for example, that $n^{\alpha}(t)>n_{\max }^{\alpha}$ and $n^{\beta}(t) \leq n_{\max }^{\beta}$. Then, there is no selection of group members that allows our agent to achieve $n^{\alpha}(t)$ signals from source $\alpha$. Thus, after choosing $n_{\max }^{\alpha}$ agents that collect $\alpha$-signals (agents chosen in the interval $\left[\underline{t}^{\alpha}, 1\right]$ ), the agent is better off choosing the remaining agents so that they collect as many $\beta$-signals as possible (this can be achieved, for instance, by choosing them in the interval $\left.\left[0, \bar{t}^{\beta}\right]\right)$. This could lead to more $\beta$-signals than in the unconstrained solution.

If both $n^{\alpha}(t)>n_{\max }^{\alpha}$ and $n^{\beta}(t)>n_{\max }^{\beta}$, the agent chooses a group in which $n_{\max }^{\alpha}$ and $n_{\max }^{\beta}$ signals from sources $\alpha$ and $\beta$ are collected, respectively. This can be achieved by selecting $n_{\max }^{\alpha}$ agents in the interval $\left[\underline{t}^{\alpha}, 1\right]$ and $n_{\max }^{\beta}$ in the interval $\left[0, \bar{t}^{\beta}\right]$.

\section{Proof of Proposition 2.}

1. For any $k=1, \ldots, n$, denote by $t(k)$ the taste parameter with which an agent is indifferent between $k-1$ and $k \alpha$-signals. That is,

$$
U(t(k), k-1, n-k+1)=U(t(k), k, n-k)
$$


It follows that an agent with taste parameter $t$ would like no $\alpha$-signals whenever $t \in[0, t(1))$, would

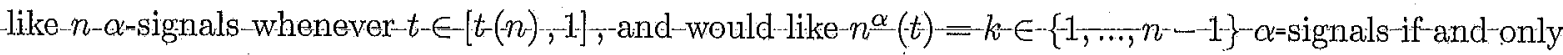
if $t \in[t(k), t(k+1))$. Since, from Proposition 1 , if $c=0$ each agent can achieve her optimal allocation of signals with some group, stability boils. down to all of the group members agreeing on the ideal number of signals of each source that are to be collected. The claim follows.

2. From the definition of $n^{\alpha}(t)$, we have $n^{\alpha}(t)=\left\lfloor m^{\alpha}(t)\right\rfloor$ if $m^{\alpha}(t) \in[0, n], n^{\alpha}(t)=0$ if $m^{\alpha}(t)<0$, and $n^{\alpha}(t)=n$ if $m^{\alpha}(t)>n$, where $m^{\alpha}(t)$ is the number achieving equality in condition (2). Simple algebraic manipulation yields:

$$
m^{\alpha}(t)=\frac{\ln \left(\frac{1}{t}-1\right)+n \ln \left(1-q_{\beta}\right)+\ln \left(1-q_{\alpha}\right)+\ln \frac{q_{\beta}}{q_{\alpha}}}{\ln \left(1-q_{\alpha}\right)+\ln \left(1-q_{\beta}\right)} .
$$

Differentiating $m^{\alpha}(t)$ we get:

$$
\begin{aligned}
& \frac{d m^{\alpha}(t)}{d t}=-\frac{1}{t(1-t) \ln \left[\left(1-q_{\alpha}\right)\left(1-q_{\beta}\right)\right]}>0 ; \\
& \frac{d^{2} m^{\alpha}(t)}{d t^{2}}=\frac{1-2 t}{[t(1-t)]^{2} \ln \left[\left(1-q_{\alpha}\right)\left(1-q_{\beta}\right)\right]} .
\end{aligned}
$$

Therefore, $m^{\alpha}(t)$ is an increasing function that is concave up to $t=\frac{1}{2}$ and convex thereafter. Since for any $k=1, \ldots, n-1, T_{k}^{n}=\left(m^{\alpha}\right)^{-1}([k, k+1))$, this implies that the sequence of intervals $\left\{T_{k}^{n}\right\}_{k=1}^{n-1}$ is such that the intervals are increasing in length until the interval $T_{\vec{k}}^{n}$ such that $1 / 2 \in T_{\vec{k}}^{n}$ and decreasing thereafter.

We now address the extreme intervals $T_{0}^{n}$ and $T_{n}^{n}$. We will show that these intervals follow the same pattern of $\left\{T_{k}^{n}\right\}_{k=1}^{n-1}$ if either $q_{\alpha}, q_{\beta}$ are high enough, or $n$ is high enough. Recall that $T_{0}^{n}=[0, t(1))$ and $T_{n}^{n}=[t(n), 1]$. From the definition of $t(k)$ above, we get

$$
t(k)=\frac{\left(1-q_{\beta}\right)^{n-k} q_{\beta}}{\left(1-q_{\alpha}\right)^{k-1} q_{\alpha}+\left(1-q_{\beta}\right)^{n-k} q_{\beta}} .
$$

We have that $t(1) \leq t(2)-t(1)$ (i.e., interval $T_{0}^{n}$ is shorter than $T_{1}^{n}$ ) if and only if

$$
\frac{2\left(1-q_{\beta}\right)^{n-1} q_{\beta}}{q_{\alpha}+\left(1-q_{\beta}\right)^{n-1} q_{\beta}} \leq \frac{\cdot\left(1-q_{\beta}\right)^{n-2} q_{\beta}}{\left(1-q_{\alpha}\right) q_{\alpha}+\left(1-q_{\beta}\right)^{n-2} q_{\beta}}
$$


Rearranging, the above condition is satisfied if and only if

$$
2 q_{\alpha}\left(1-q_{\alpha}\right)\left(1-q_{\beta}\right)+\left(1-q_{\beta}\right)^{n-1} q_{\beta} \leq q_{\alpha}
$$

Condition (10) is a necessary and sufficient condition on $q_{\alpha}, q_{\beta}$, and $n$ such that the interval $T_{0}^{n i}$ follows the same pattern of the sequence $\left\{T_{k}^{n}\right\}_{k=1}^{n-1}$. Note that, since $(1-x) x$ is maximized in $\left[\frac{1}{2}, 1\right]$ at $x=1 / 2$, $q_{\alpha}, q_{\beta} \geq \frac{1}{2}$ is a sufficient condition to guarantee (10), as we have

$$
2\left(1-q_{\alpha}\right) q_{\alpha}\left(1-q_{\beta}\right)+\left(1-q_{\beta}\right)^{n-1} q_{\beta} \leq\left(1-q_{\alpha}\right) q_{\alpha}+\frac{1}{4} \leq \frac{1}{2} \leq q_{\alpha}
$$

Moreover, if $\left(1-q_{\alpha}\right)\left(1-q_{\beta}\right)<1 / 2$, condition (10) holds for $n$ large enough.

The interval $T_{n}^{n}$ is shorter than $T_{n-1}^{n}$ if and only if $1-t(n) \leq t(n)-t(n-1)$. After rearranging, this is equivalent to

$$
2 q_{\beta}\left(1-q_{\alpha}\right)\left(1-q_{\beta}\right)+\left(1-q_{\alpha}\right)^{n-1} \cdot q_{\alpha} \leq q_{\beta} .
$$

As before, it is easy to see that if $\left(1-q_{\alpha}\right)\left(1-q_{\beta}\right)<1 / 2$, condition (11) is satisfied for large enough $n$, and that $q_{\alpha}, q_{\beta} \geq \frac{1}{2}$ is a sufficient condition for (11) to be satisfied for any $n$.

Proof of Proposition 3A. Suppose $n>n_{\max }^{\alpha}+n_{\max }^{\beta}$. Any agent of taste $t=0$ is in an optimal group as long as there are $n_{\max }^{\alpha}$ agents who are acquiring an $\alpha$-signal. Similarly, any agent of taste $t=1$ is in an optimal group as long as there are $n_{\max }^{\beta}$ agents who are acquiring a $\beta$-signal. Any agent with $t \in(0,1)$ is in an optimal group as long as there are $n_{\max }^{\alpha}$ and $n_{\max }^{\beta}$ agents acquiring an $\alpha$ - and $\beta$-signal, respectively (indeed, she can contemplate a group with $n_{\max }^{\alpha}$ and $n_{\max }^{\beta}$ agents of taste $t=1$ and $t=0$, respectively). Therefore; stable groups take one of the forms (1) or (2).

Proof of Lemma 2. Suppose that $n^{\alpha}(t)>n_{c}^{\alpha}(t)$. Then, it must be the case that $n^{\beta}(t)<n_{c}^{\beta}(t)$ (otherwise, $n=n^{\alpha}(t)+n^{\beta}(t)>n_{c}^{\alpha}(t)+n_{c}^{\beta}(t)=n_{c}(t)$, contrary to our assumption). That is, $n_{c}^{\beta}(t) \geq$ $n^{\beta}(t)+1$. In particular,

$$
\frac{1-t}{2}\left[1-\left(1-q_{\beta}\right)^{n^{\beta}(t)+1}\right]-\frac{1-t}{2}\left[1-\left(1-q_{\beta}\right)^{n^{\beta}(t)}\right] \geq c
$$


From the definition of $n^{\alpha}(t), n^{\beta}(t)$,

$$
\begin{aligned}
& \frac{t}{2}\left[1-\left(1-q_{\alpha}\right)^{n^{\alpha}(t)}\right]+\frac{1-t}{2}\left[1-\left(1-q_{\beta}\right)^{n^{\beta}(t)}\right] \\
\geq & \frac{t}{2}\left[1-\left(1-q_{\alpha}\right)^{n^{\alpha}(t)-1}\right]+\frac{1-t}{2}\left[1-\left(1-q_{\beta}\right)^{n^{\beta}(t)+1}\right],
\end{aligned}
$$

which is equivalent to

$$
\begin{aligned}
& t\left[1-\left(1-q_{\alpha}\right)^{n^{\alpha}(t)}\right]-\left[1-\left(1-q_{\alpha}\right)^{n^{\alpha}(t)-1}\right] \\
\geq & (1-t)\left[1-\left(1-q_{\beta}\right)^{n^{\beta}(t)+1}\right]-(1-t)\left[1-\left(1-q_{\beta}\right)^{n^{\beta}(t)}\right] \geq c
\end{aligned}
$$

and $n_{c}^{\alpha}(t) \geq n^{\alpha}(t)$, which contradicts our hypothesis. Identical arguments follow if $n^{\beta}(t)>n_{c}^{\beta}(t)$.

Analysis of $\mathcal{L}(t)$. The shape of $n_{c}(t)$ plays a crucial role in our discussion. As described in the text, we define $m_{c}^{\alpha}$ and $m_{c}^{\beta}$ as the real numbers achieving equality in (3) and (4), respectively, so that

$$
\begin{aligned}
m_{c}^{\alpha}(t) & \doteq \frac{\ln c-\ln t-\ln q_{\alpha}-\ln 2}{\ln \left(1-q_{\alpha}\right)}+1 \\
m_{c}^{\beta}(t) & \equiv \frac{\ln c-\ln (1-t)-\ln q_{\beta}-\ln 2}{\ln \left(1-q_{\beta}\right)}+1
\end{aligned}
$$

and $n_{c}^{x}(t)=\left\lfloor m_{c}^{x}(t)\right\rfloor$ if $m_{c}^{x}(t) \geq 0$ and $n_{c}^{x}(t)=0$ if $m_{c}^{x}(t)<0$ for $x=\alpha, \beta$.

Assume $c$ is sufficiently small so that $\frac{c\left(1-q^{\alpha}\right)}{t q^{\alpha}} \leq 1$ and $\frac{c\left(1-q^{\beta}\right)}{(1-t) q^{\beta}} \leq 1$, i.e., $n_{c}^{\alpha}(t), n_{c}^{\beta}(t)>0$, for all $t$. Ignoring rounding, the relevant function for studying $n_{c}(t)$ is:

$$
\begin{aligned}
\mathcal{L}(t) & =\max \left\{m_{c}^{\alpha}(t), 0\right\}+\max \left\{m_{c}^{\beta}(t), 0\right\}= \\
& =\frac{\ln c-\ln t-\ln q^{\alpha}-\ln 2}{\ln \left(1-q^{\alpha}\right)}+\frac{\ln c-\ln (1-t)-\ln q^{\beta}-\ln 2}{\ln \left(1-q^{\beta}\right)}+2 .
\end{aligned}
$$

The unconstrained maximizing $\hat{t}$ is given by:

$$
\frac{1-\hat{t}}{\hat{t}}=\frac{\ln \left(1-q^{\alpha}\right)}{\ln \left(1-q^{\beta}\right)} \Leftrightarrow \hat{t}=\frac{\ln \left(1-q^{\beta}\right)}{\ln \left(1-q^{\alpha}\right)+\ln \left(1-q^{\beta}\right)} .
$$

$\hat{t}$. is the taste parameter maximizing $\mathcal{L}(t)$. 
Consider the derivative of $\mathcal{L}(t)$ :

$$
\mathcal{L}^{\prime}(t)=\frac{1}{(1-t)} \frac{1}{\ln \left(1-q^{\beta}\right)}-\frac{1}{t} \frac{1}{\ln \left(1-q^{\alpha}\right)}
$$

Notice that it is positive up to $\hat{t}$ and negative afterwards. Indeed, $\mathcal{L}(t)$ is concave.

When there exists a $t$ for which $\frac{c\left(1-q^{\alpha}\right)}{t q^{\alpha}} \leq 1$ and $\frac{c\left(1-q^{\beta}\right)}{(1-t) q^{\beta}}>1$, these conditions hold within $[0, t]$, and $\mathcal{L}(t)$ takes the form

$$
\mathcal{L}(t)=m_{c}^{\alpha}(t)=\frac{\ln c-\ln t-\ln q^{\alpha}-\ln 2}{\ln \left(1-q^{\alpha}\right)}+1
$$

which is decreasing and concave. Similar analysis pertains to ranges for which $\frac{c\left(1-q^{\alpha}\right)}{t q^{\alpha}}>1$ and $\frac{c\left(1-q^{\beta}\right)}{(1-t) q^{\beta}} \leq 1$.

Proposition 3C If $n=n_{\max }^{\alpha}+n_{\max }^{\beta}$, stable groups take one of the following forms:

1. $n_{\max }^{\alpha}$ agents whose taste falls in $\left[\underline{t}^{\alpha}, 1\right]$ and $n_{\max }^{\beta}$ agents whose taste falls in $\left[0, \bar{t}^{\beta}\right]$;

2. $n$ agents of taste $t=1$, or $n$ agents of taste $t=0$;

3. If $n_{\max }^{\alpha}=1$, then $n_{\max }^{\beta}$ agents of taste 0 , and one agent of taste $t \in(0,1)$, where $t$ satisfies one of the following:

(a) $n_{c}^{\alpha}(t)=0$ and $U\left(t, 0, n_{\max }^{\beta}\right) \geq U\left(t, 1, n_{\max }^{\beta}-1\right)$; or

(b) $n^{\alpha}(t)=n_{c}^{\alpha}(t)=1$.

Similarly if $n_{\max }^{\beta}=1$.

Proof of Proposition 3C. The analysis in Proposition $3 \mathrm{~A}$ carries through as long as $n_{\max }^{\alpha}, n_{\max }^{\beta}>1$, and the classes of group compositions in points (1) and (2) constitute all of the stable allocations.

Regarding (3), suppose that $n_{\max }^{\alpha}=1$. (a) Assume that $n_{c}^{\alpha}(t)=0$. First, consider $t$ for which $n^{\alpha}(t)=n_{c}^{\alpha}(t)=0$. The group consisting of an agent of type $t$ and $n_{\max }^{\beta}$ agents of taste 0 is stable, since the agent with non-extreme taste parameter $t$ does not have enough incentives to get information on one issue, even when she is the first to acquire a signal relevant to it. Moreover, the remaining agents have an extreme taste parameter, so have no incentive to acquire a signal other than the one 
pertaining to the issue they care most about. In this case, we have

$$
U\left(t, 0, n_{\max }^{\beta}\right)-U\left(t, 1, n_{\max }^{\beta}-1\right) \geq U\left(t, 0, n_{\max }^{\beta}+1\right)-U\left(t, 1, n_{\max }^{\beta}\right) \geq 0
$$

where the last inequality follows from $n^{\alpha}(t)=0$.

Second, consider the case in which $n_{c}^{\alpha}(t)=0$ and $n^{\alpha}(t)=1$. The group is stable since the condition $U\left(t, 0, n_{\max }^{\beta}\right) \geq U\left(t, 1, n_{\max }^{\beta}-1\right)$ assures that the utility the agent gets from the $n_{\max }^{\beta}$-th $\beta$-signal is higher (or equal) to the utility she would get from the first $\alpha$-signal.

(b) Suppose $n^{\alpha}(t)=n_{c}^{\alpha}(t)=1$. In this case, the group formed by $n_{\max }^{\beta}$ agents of taste $t=0$ and one agent of type $t$ is stable as the agent of taste $t$ implements her unconstrained optimal allocation.

Analogous constructions can be performed when $n_{\max }^{\beta}=1$.

\section{Proof of Proposition 4.}

1. To stress the comparative statics on $n$, let $x_{f}^{\alpha}(n, t) \equiv m^{\alpha}(t)$ with $m^{\alpha} \cdot(t)$, as defined before, for group size $n$. Then, for any $t \in[0,1]$, we have

$$
\begin{aligned}
x_{f}^{\alpha}(n, t) & =\frac{\ln \left(\frac{1}{t}-1\right)+n \ln \left(1-q_{\beta}\right)+\ln \left(1-q_{\alpha}\right)+\ln \frac{q_{\beta}}{q_{\alpha}}}{\ln \left(1-q_{\alpha}\right)+\ln \left(1-q_{\beta}\right)} \\
& =A+B n+C \ln \left(\frac{1}{t}-1\right),
\end{aligned}
$$

where $A=\frac{\ln \left(1-q_{\alpha}\right)+\ln \frac{q_{\beta}}{q_{\alpha}}}{\ln \left(1-q_{\alpha}\right)+\ln \left(1-q_{\beta}\right)}, B=\frac{\ln \left(1-q_{\beta}\right)}{\ln \left(1-q_{\alpha}\right)+\ln \left(1-q_{\beta}\right)}$, and $C=\left(\ln \left(1-q_{\alpha}\right)+\ln \left(1-q_{\beta}\right)\right)^{-1}$. Consider $t^{\prime}$, $t^{\prime \prime}$; and $n$ such that $t^{\prime}, t^{\prime \prime} \in T_{k}^{n}$ for some $k=1, . ., n-1$. From the definition of $\left\{T_{k}^{n}\right\}_{k=1}^{n-1}$, this implies that $\left\lfloor x_{f}^{\alpha}\left(n, t^{\prime}\right)\right\rfloor=\left\lfloor x_{f}^{\alpha}\left(n, t^{\prime \prime}\right)\right\rfloor$, so that $t^{\prime}, t^{\prime \prime} \in T_{\left\lfloor x_{f}^{\alpha}\left(n, t^{\prime}\right)\right\rfloor}^{n}$ :

First, we show that there exists $\widehat{n}>n$ such that $t^{\prime}, t^{\prime \prime} \in T_{h}^{\widehat{n}}$ for some $h=1, . ., \widehat{n}-1$. Let $\Delta=$ $\max \left\{x\left(t^{\prime}, n\right)-\left\lfloor x\left(t^{\prime}, n\right)\right\rfloor, x\left(t^{\prime \prime}, n\right)-\left\lfloor x\left(t^{\prime \prime}, n\right)\right\rfloor\right\}$. That is, $\Delta$ is the maximal distance between $x\left(t^{\prime}, n\right)$ and $x\left(t^{\prime \prime}, n\right)$ and their rounding down to an integer. Let $r$ be an integer such that $r B-\lfloor r B\rfloor<1-\Delta$. Therefore, for the integer $d \equiv\lfloor r B\rfloor$, we have $B \in\left[\frac{d}{r}, \frac{d+1-\Delta}{r}\right)$. Define $\hat{n}=n+r$. Notice that

$$
\begin{aligned}
& x\left(t^{\prime}, \hat{n}\right) \in\left[x\left(t^{\prime}, n\right)+d, x\left(t^{\prime}, n\right)+d+1-\Delta\right) \\
& x\left(t^{\prime \prime}, \hat{n}\right) \in\left[x\left(t^{\prime \prime}, n\right)+d, x\left(t^{\prime \prime}, n\right)+d+1-\Delta\right)
\end{aligned}
$$


From the definition of $\Delta$, it follows that $\left\lfloor x\left(t^{\prime}, \hat{n}\right)\right\rfloor=\left\lfloor x\left(t^{\prime \prime}, \hat{n}\right)\right\rfloor=\left\lfloor x\left(t^{\prime}, n\right)\right\rfloor+d$. Thus, $t^{\prime}, t^{\prime \prime} \in$

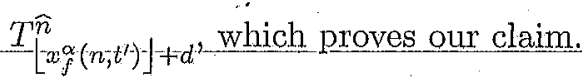

Moreover, note that, if $t^{\prime}>t^{\prime \prime}, x_{f}^{\alpha}\left(n, t^{\prime}\right)-x_{f}^{\alpha}\left(n, t^{\prime \prime}\right)=C\left[\ln \left(\frac{1}{t^{\prime}}-1\right)-\ln \left(\frac{1}{t^{\prime \prime}}-1\right)\right]<1$. Thus, for any $n^{\prime}, x_{f}^{\alpha}\left(n^{\prime}, t^{\prime}\right)-x_{f}^{\alpha}\left(n^{\prime}, t^{\prime \prime}\right)=x_{f}^{\alpha}\left(n, t^{\prime}\right)-x_{f}^{\alpha}\left(n ; t^{\prime \prime}\right)<1$. This implies that for any $n^{\prime}$, either $t^{\prime}, t^{\prime \prime} \in T_{h}^{n^{\prime}}$, or $t^{\prime} \in T_{h+1}^{n^{\prime}}$ and $t^{\prime \prime} \in T_{h}^{n^{\prime}}$ for some $h$. That is, if $t^{\prime}, t^{\prime \prime}$ are two táste parameters for which there is agreement on the optimal number of $\alpha$-signals in $\{1, . ., n-1\}$ for a group of size $n$, then even if they disagree for some $n^{\prime}>n$, such disagreement can pertain to the allocation of at most one signal.

2. Observe that the interval $T_{0}^{n}$ contains all $t$ such that $t<\frac{\left(1-q_{\beta}\right)^{n-1} q_{\beta}}{q_{\alpha}+\left(1-q_{\beta}\right)^{n-1} q_{\beta}}=t^{n}(1)$. It follows that $T_{0}^{n^{\prime}} \subsetneq T_{0}^{n}$ for any $n^{\prime}>n$ and, for any $q_{\alpha}, q_{\beta} \in(0,1), t^{n}(1) \searrow_{n \rightarrow \infty} 0$. so the interval $T_{0}^{n}$ shrinks to a singleton as the size of the group becomes infinitely large. Similarly, $T_{n}^{n}$ contains all $t \geq \frac{q_{\beta}}{\left(1-q_{\alpha}\right)^{n-1} q_{\alpha}+q_{\beta}}=t^{n}(n)$. It follows that $T_{n^{\prime}}^{n^{\prime}} \subsetneq T_{n}^{n}$ for any $n^{\prime}>n$ and, for any $q_{\alpha}, q_{\beta} \in(0,1)$, $t^{n}(n) \nearrow_{n \rightarrow \infty} 1$, so the interval $T_{n}^{n}$ shrinks to a singleton as the size of the group becomes infinitely large as well.

\section{Proof of Lemma 3.}

1. Recall that $\left\{t_{1}, . ., t_{r}\right\}$ is the set of taste parameters $t_{i}$ such that there is at least one agent in $N$ with taste $t_{i}$. Let any group of agents $G \subseteq N$ be identified by a vector $\left(z_{1}, \ldots, z_{r}\right)$, where $z_{l} \leq m_{l}$ is the number of agents of taste $t_{l}$ in group $G$. Suppose that $\mathcal{G}=\left\{G_{1}, . ., G_{s}\right\}$ is a stable allocation, and let $t_{i}, t_{j}, t_{h} \in\left\{t_{1}, . ., t_{r}\right\}$ be such that $t_{i}>t_{j}>t_{h}$, and suppose that two agents with tastes $t_{i}$ and $t_{h}$, respeotively, are in a group $G \in \mathcal{G}$ and that an agent with taste $t_{j}$ is in $G^{\prime} \neq G$. Assume that in any equilibrium of the information-collection game, $k^{\alpha} \alpha$-signals and $k^{\beta} \beta$-signals are acquired in group $G$, and $k^{\alpha \prime} \alpha$-signals and $k^{\beta \prime} \beta$-signals are acquired in group $G^{\prime}$. Since $t_{j}$ at least weakly prefers $G^{\prime}$ over $G$ it must be the case that either $k^{\alpha}<k^{\alpha \prime}$ or $k^{\beta}<k^{\beta \prime}$. Suppose $k^{\alpha} \cdot<k^{\alpha \prime}$. Certainly, if $k^{\beta} \leq k^{\beta \prime}$, the other two agents. (of types $t_{i}$ and $t_{h}$ ) would benefit by switching to group $G^{\prime}$. Assume then that $k^{\beta}>k^{\beta \prime}$. Optimality for the agent of type $t_{j}$ then implies:

$$
\begin{aligned}
& t_{j}\left(1-\left(1-q_{\alpha}\right)^{k^{\alpha \prime}}\right)+\left(1-t_{j}\right)\left(1-\left(1-q_{\beta}\right)^{k^{\beta \prime}}\right) \\
\geq & t_{j}\left(1-\left(1-q_{\alpha}\right)^{k^{\alpha}}\right)+\left(1-t_{j}\right)\left(1-\left(1-q_{\beta}\right)^{k^{\beta}}\right)
\end{aligned}
$$




$$
\Longleftrightarrow \frac{t_{j}}{1-t_{j}} \geq \frac{\left(1-q_{\beta}\right)^{k^{\beta \prime}}-\left(1-q_{\beta}\right)^{k^{\beta}}}{\left(1-q_{\alpha}\right)^{k^{\alpha}}-\left(1-q_{\alpha}\right)^{k^{\alpha \prime}}} .
$$

However, since $\frac{t_{h}}{1-t_{h}}>\frac{t_{j}}{1-t_{j}}$ and the agent of type $t_{h}$ joining $G^{\prime}$ would imply an additional signal collected, it follows that an agent of type $t_{h}$ would strictly benefit by shifting from group $G$ to group $G^{\prime}$, in contradiction. The case $k^{\beta}<k^{\beta \prime}$ follows analogously.

2. Suppose that $\mathcal{G}$ is a stable allocation, and suppose that $G, G^{\prime} \in \mathcal{G}, G \neq G^{\prime}$ both contain at least one agent of taste $t_{i}$. If one group identified by $\left(x_{1}, . ., x_{r}\right)$ is (weakly) preferred by an agent of taste $t_{i}$ to the group identified by $\left(x_{1}^{\prime}, \ldots, x_{r}^{\prime}\right)$, we write $\left(x_{1}, \ldots, x_{r}\right) \succcurlyeq t_{i}\left(x_{1}^{\prime}, \ldots, x_{r}^{\prime}\right)$. Assume that $G$ is identified by $\left(x_{1}, . ., x_{i}, . . x_{r}\right)$ and that $G^{\prime}$ is identified by $\left(y_{1}, . ., y_{i}, . ., y_{r}\right)$. For an agent of taste $t_{i}$, a deviation from $G$ to $G^{\prime}$ is unprofitable if

$$
\left(x_{1}, . ., x_{i}, \ldots x_{r}\right) \succcurlyeq_{t_{i}}\left(y_{1}, . ., y_{i}+1, . ., y_{r}\right)
$$

Similarly, for an agent of taste $t_{i}^{\prime}$, a deviation from $G^{\prime}$ to $G$ is unprofitable if

$$
\left(y_{1}, . ., y_{i}, . ., y_{r}\right) \succcurlyeq_{t_{i}}\left(x_{1}, . ., x_{i}+1, . . x_{r}\right)
$$

However, any agent of taste $t_{i}$ strictly benefits from having her group augmented by one more member of her own type. Since $\left(z_{1}, . . z_{s}+1, \ldots, z_{r}\right) \succ_{t_{s}}\left(z_{1}, . . z_{s}, \ldots, z_{r}\right)$, for any $\left(z_{1}, . . z_{s}, \ldots, z_{r}\right)$ and $t_{s}$, we get a contradiction.

Proof of Proposition 5. For $x=\alpha, \beta$, denote by $z^{x}(t, h) \equiv n^{x}(t)$ the optimal number of $x$-signals out of a total of $h$ signals for an agent of taste $t$. Moreover, for any $t_{1}, t_{2} \in[0,1]$, let $w^{x}\left(t_{1}, t_{2}, h_{1}, h_{2}\right)$ denote the equilibrium number of $x$-signals collected in a group that is composed of $h_{1}$ agents of taste $t_{1}$ and $h_{2}$ agents of taste $t_{2}$ (well-defined from Lemma 1A).

1. Observe that if $z^{\alpha}\left(t_{1}, N\right)>z^{\alpha}\left(t_{2}, N\right)$ then either $w^{\alpha}\left(t_{1}, t_{2}, 1, N\right)=z^{\alpha}\left(t_{2}, N\right)+1$ (if $z^{\alpha}\left(t_{2}, N+\right.$ $\left.1)=z^{\alpha}\left(t_{2}, N\right)+1\right)$, or $w^{\alpha}\left(t_{1}, t_{2}, 1, N\right)=z^{\alpha}\left(t_{2}, N\right)$ (if $z^{\alpha}\left(t_{2}, N+1\right)=z^{\alpha}\left(t_{2}, N\right)$ ).

Consider a fully segregated partition and suppose that agent $a \in N_{i}$ has taste parameter $t_{i}$ for $i, \in\{1, . ., r\}$. Since $\left|N_{i}\right|=m$ for all $i$, checking that such an agent does not have a profitable deviation by joining $N_{i+1} \cup\{a\}$ and $N_{i-1} \cup\{a\}$ is enough to guarantee that this agent does not have profitable deviations (note that for $i=1, r$, there is only one constraint to check). Consider a deviation of 
agent $a$ from $N_{i}$ to $N_{i+1} \cup\{a\}$. Since $t_{i} \geq t_{i+1}, z^{\alpha}\left(t_{i}, n\right) \geq z^{\alpha}\left(t_{i+1}, n\right)$ for all $n \geq 1$. A necessary condition for the deviation not to be strictly beneficial is that $z^{\alpha}\left(\underline{t}_{i}, \underline{m}\right) \geq z^{\alpha}\left(t_{i+1}, m\right)$.... Suppose first that $w^{\alpha}\left(t_{i}, t_{i+1}, 1, m\right)=z^{\alpha}\left(t_{i+1}, m\right)+1$. It follows that the deviation is not profitable whenever

$$
\begin{aligned}
& t_{i}\left[1-\frac{1}{2}\left(1-q_{\alpha}\right)^{z^{\alpha}\left(t_{i}, m\right)}\right]+\left(1-t_{i}\right)\left[1-\frac{1}{2}\left(1-q_{\beta}\right)^{m-z^{\alpha}\left(t_{i}, m\right)}\right] \\
\geq & t_{i}\left[1-\frac{1}{2}\left(1-q_{\alpha}\right)^{z^{\alpha}\left(t_{i+1}, m\right)+1}\right]+\left(1-t_{i}\right)\left[1-\frac{1}{2}\left(1-q_{\beta}\right)^{m-z^{\alpha}\left(t_{i+1}, m\right)}\right],
\end{aligned}
$$

or, rearranging terms,

$$
t_{i}\left[\left(1-q_{\alpha}\right)^{z^{\alpha}\left(t_{i+1}, m\right)+1}-\left(1-q_{\alpha}\right)^{z^{\alpha}\left(t_{i}, m\right)}\right] \geq\left(1-t_{i}\right)\left[\left(1-q_{\beta}\right)^{m-z^{\alpha}\left(t_{i}, m\right)}-\left(1-q_{\beta}\right)^{m-z^{\alpha}\left(t_{i+1}, m\right)}\right]
$$

For $x=\alpha, \beta$, define $v^{x}(k) \equiv\left(1-q_{x}\right)^{k}-\left(1-q_{x}\right)^{k+1}$; so that $v^{x}(k)$ is the marginal contribution of the $(k+1)$ 'th $x$-signal for $x=\alpha, \beta$ (up to a factor of $\frac{1}{2}$ ). Note that, for $x=\alpha, \beta, v^{x}(k)$ is decreasing in $k$. Thus, substituting and rearranging terms, we can rewrite (13) as follows:

$$
\frac{t_{i}}{1-t_{i}} \geq \frac{\sum_{k=m-z^{\alpha}\left(t_{i}, m\right)}^{m-z^{\alpha}\left(t_{i+1}, m\right)-1} v^{\beta}(k)}{\sum_{z^{\alpha}\left(t_{i}, m\right)-1} v^{\alpha}\left(t_{i+1}, m\right)+1}
$$

where we use the convention that $\sum_{k=w}^{w-1} v^{\alpha}(k) \equiv 0$ for any $w$. Condition (14) implicitly defines a condition on $t_{i}$ and $t_{i+1}$ for a deviation from $N_{i}$ to $N_{i+1}$ to be unprofitable.

Observe that, if $w^{\alpha}\left(t_{i}, t_{i+1}, 1, m\right)=z^{\alpha}\left(t_{i+1} ; m\right)$, a deviation of agent $a$ of taste parameter $t_{i}$ to a group of $m$ agents of taste parameter $\dot{t}_{i+1}$ is less profitable than a deviation to a group in which $z^{\alpha}\left(t_{i+1}, m\right)+1$ out of $m+1$ agents collect the $\alpha$-signal, and therefore, condition (14) is sufficient to guarantee that such deviation is not profitable.

If $t_{i}$ is fixed, condition (14) is weaker the lower is $t_{i+1}$ (that is, the further apart $t_{i}$ and $t_{i+1}$ are). This guarantees that there exists $\underline{t}\left(t_{i}\right)$ such that a deviation of agent $a$ in $N_{i}$ to $N_{i+1}$ is unprofitable if and only if $t_{i+1}<\underline{t}\left(t_{i}\right)$. If $t_{i+1} \in T_{0}^{m}$ and condition (14) is not satisfied, then $\underline{t}\left(t_{i}\right)=0$. Set $\underline{t}\left(t_{i}\right)=0$ if $z^{\alpha}\left(t_{i}, m\right)=z^{\alpha}\left(t_{i+1}, m\right)=0$. 
We can follow a similar procedure by considering a deviation from $N_{i}$ to $N_{i-1} \cup\{a\}$ and defining a taste $\bar{t}\left(\underline{t}_{i}\right)$ such that a deviation from $N_{i}$ to $N_{i=1} \cup\{\underline{a}\}$ is unprofitable if and only if $t_{i=1}>\dot{\bar{t}}\left(t_{i}\right)$. The intervals $\left\{\mathcal{T}_{i}\right\}_{i=1}^{r}$ are obtained by setting for any $i \in\{1, \ldots, r\} ; \mathcal{T}_{i} \equiv\left[\underline{t}\left(t_{i}\right), \bar{t}\left(t_{i}\right)\right)$ whenever $\bar{t}\left(t_{i}\right)<$ 1 and $\mathcal{T}_{i} \equiv\left[\underline{t}\left(t_{i}\right), 1\right]$ whenever $\vec{t}\left(t_{i}\right)=1$. To see that $\mathcal{T}_{i}$ are unions of contiguous intervals of the sequence $\left\{T_{k}^{m}\right\}_{k=1}^{m}$, observe that all $t_{i+1}$ in the same interval $T_{k}^{m}$ share the same optimal allocation $\left(z^{\alpha}\left(t_{i+1}, m\right), z^{\beta}\left(t_{i+1}, m\right)\right)$. Thus, for a given $t_{i}$, if condition (14) is satisfied for $t_{i+1} \in T_{k}^{m}$, then it is satisfied for any $t_{i+1}^{\prime} \in T_{k}^{m}$.

2. For each $i$, denote $\mathcal{T}_{i}^{-}=\mathcal{T}_{i} \cap\left[0, t_{i}\right]$ and $\mathcal{T}_{i}^{+}=\mathcal{T}_{i} \cap\left[t_{i}, 1\right]$ the sub-intervals of $\mathcal{T}_{i}$ that are to the left and right of $t_{i}$, respectively. Notice that for sufficiently low $t_{i}, \mathcal{T}_{i}^{-}=\left[0, t_{i}\right]$, the length of which is increasing in $t_{i}$ (and decreasing in $i$ ). Similarly, for sufficiently high $t_{i}, \mathcal{T}_{i}^{+}=\left[t_{i}, 1\right]$, the length of which is decreasing in $t_{i}$ (and increasing in $i$ ). To show the claim, it suffices to illustrate that for sufficiently low $t_{i}, \mathcal{T}_{i}^{+}, \ldots, \mathcal{T}_{s}^{+}$are decreasing in length and, similarly, for sufficiently high $t_{i}, \mathcal{T}_{1}^{-}, \ldots, \mathcal{T}_{i}^{-}$ are increasing in length.

Consider $t$ and $t^{\prime}$, with $t^{\prime}>t$, and $k \in\left\{1, . ., z^{\alpha}(t, m)\right\}$, such that the agent of type $t$ prefers to stay in her group collecting $z^{\alpha}(t, m) \alpha$-signals than to be the $m+1$ 'th member of a group in which, without her, $z^{\alpha}(t, m)-k \alpha$-signals are collected. Upon joining such a group, the agent would be collecting an $\alpha$-signal, and so the corresponding incentive constraint (similar to condition (13)) implies that:

$$
\frac{t}{1-t} \geq \frac{\left(1-q_{\beta}\right)^{m-z^{\alpha}(t, m)}-\left(1-q_{\beta}\right)^{m-z^{\alpha}(t, m)+k}}{\left(1-q_{\alpha}\right)^{z^{\alpha}(t, m)-k+1}-\left(1-q_{\alpha}\right)^{z^{\alpha}(t, m)}}
$$

Let

$$
\begin{aligned}
A(t) & \equiv t\left[\left(1-q_{\alpha}\right)^{m^{\alpha}(t)-k+1}-\left(1-q_{\alpha}\right)^{m^{\alpha}(t)}\right] \text { and } \\
B(t) & \equiv(1-t)\left[\left(1-q_{\beta}\right)^{m-m^{\alpha}(t)}-\left(1-q_{\beta}\right)^{m-m^{\alpha}(t)+k}\right]
\end{aligned}
$$

and recall that

$$
m^{\alpha}(t)=\frac{\ln \left(\frac{1}{t}-1\right)+m \ln \left(1-q_{\beta}\right)+\ln \left(1-q_{\alpha}\right)+\ln \frac{q_{\beta}}{q_{\alpha}}}{\ln \left(1-q_{\alpha}\right)+\ln \left(1-q_{\beta}\right)} .
$$

Note that whenever $z^{\alpha}(t, m)=z^{\alpha}\left(t^{\prime}, m\right)$, if (15) holds for $t$, it will hold for $t^{\prime}$. In order to show the claim, it is therefore sufficient to focus on $t$ and $t^{\prime}$ that are at the cusps of our original intervals 
$\left\{T_{k}^{m}\right\}_{k}$, for which $m^{\alpha}(t)=z^{\alpha}(t, m)$ and $m^{\alpha}\left(t^{\prime}\right)=z^{\alpha}\left(t^{\prime}, m\right)$. For such $t$ and $t^{\prime},(15)$ is satisfied whenever $A(t) \geq B(t)$-and $-A\left(t^{\prime}\right) \geq B\left(t^{\prime}\right)$, respectively

From the derivations in the proof of Proposition 2, recall that

$$
\frac{d m^{\alpha}(t)}{d t}=-\frac{1}{t(1-t) \ln \left[\left(1-q_{\alpha}\right)\left(1-q_{\beta}\right)\right]}>0
$$

Now, we have

$$
A^{\prime}(t)=\left[\left(1-q_{\alpha}\right)^{m^{\alpha}(t)-k+1}-\left(1-q_{\alpha}\right)^{m^{\alpha}(t)}\right]\left[1+t \ln \left(1-q_{\alpha}\right) \frac{d m^{\alpha}(t)}{d t}\right]
$$

which implies that $A^{\prime}(t)$ is positive if $1+t \ln \left(1-q_{\alpha}\right) \frac{d m^{\alpha}(t)}{d t}>0$, which, after manipulation, is equivalent to

$$
t<\frac{\ln \left(1-q_{\beta}\right)}{\ln \left(1-q_{\alpha}\right)+\ln \left(1-q_{\beta}\right)}
$$

Similarly, we have

$$
B^{\prime}(t)=\left[\left(1-q_{\beta}\right)^{m-m^{\alpha}(t)}-\left(1-q_{\beta}\right)^{m-m^{\alpha}(t)+k}\right]\left[-1-(1-t) \ln \left(1-q_{\beta}\right) \frac{d m^{\alpha}(t)}{d t}\right]
$$

As before, $B^{\prime}(t)$ positive if $1+(1-t) \ln \left(1-q_{\beta}\right) \frac{d m^{\alpha}(t)}{d t}<0$, or

$$
t<\frac{\ln \left(1-q_{\beta}\right)}{\ln \left(1-q_{\alpha}\right)+\ln \left(1-q_{\beta}\right)}
$$

Notice that $A(0)=0$ and $A(1)=\left[\left(1-q_{\alpha}\right)^{m-k+1}-\left(1-q_{\alpha}\right)^{m}\right]>0$, while $B(0)=\left(1-q_{\beta}\right)^{m}$ $\left(1-q_{\beta}\right)^{m+k}>0$ and $B(1)=0$. Since both $A(t)$ and $B(t)$ are decreasing for any $t>\frac{\ln \left(1-q_{\beta}\right)}{\ln \left(1-q_{\alpha}\right)+\ln \left(1-q_{\beta}\right)}$ and $A(1)>B(1)$, it must be the case that for $t$ high enough, $A(t) \geq B(t)$ implies $A\left(t^{\prime}\right) \geq B\left(t^{\prime}\right)$ whenever $t^{\prime}>t$ (in addition, it is easy to show that both $A(t)$ and $B(t)$ are concave; therefore, they can cross at most once for $\left.t>\frac{\ln \left(1-q_{\beta}\right)}{\ln \left(1-q_{\alpha}\right)+\ln \left(1-q_{\beta}\right)}\right)$.

From the comparative statics of Proposition 2, it follows that for any $t_{i}$ high enough, $\mathcal{T}_{1}^{-}, \ldots, \mathcal{T}_{i}^{-}$ are increasing in length, as was required. The case of low $t_{i}$ follows analogously. 


\section{REFERENCES}

Benhabib, J., A. Bisin, and M. O. Jackson (editors) (2010), The Handbook of Social Economics, Elsevier.

Bramoullé, Y. and B. W. Rogers (2010), "Diversity and Popularity in Social Networks," mimeo.

Calvó-Armengol A., J. De Martí and A. Prat (2009), "Endogenous Communication in Complex Organizations," mimeo.

Che, Y-K. and N. Kartik (2009), "Opinions as Incentives," The Journal of Political Economy, 117(5), 815-860. mimeo.

Coleman, J. (1966), Medical Innovation: A Diffusion Study, Second Edition, Bobbs-Merrill. New York.

Conley, T., and C. Udry (2008), "Learning About a New Technology: Pineapple in Ghana," forthcoming on The American Economic Review.

Currarini, S., M. O. Jackson, and P. Pin (2009), "An Economic Model of Friendship: Homophily, Minorities and Segregation," Econometrica, 77(4), 1003-1045.

Demange, G. (1994), "Intermediate Preferences and Stable Coalition Structures," Journal of Mathematical Economics, 23, 45-58.

Dunbar, R. I. M. (1992), Neocortex size as a constraint on group size in primates, Journal of Human Evolution, 22, 469-493.

Ellickson, B., B. Grodal, S. Scotchmer, and W. R. Zame (1999), "Clubs and the Market," Econometrica, 67, 1185-1218.

Evans, W. N., W. E. Oates, and R. M. Schwab (1992), "Measuring Peer Group Effects: A Study of Teenage Behavior," The Journal of Political Economy, 100(5), 966-991.

Foster, A. D. and M. R. Rosenzweig (1995), "Learning by Doing and Learning from Others: Human Capital and Technical Change in Agriculture," The Journal of Political Economy, 103(6), 1176-1209.

Gerardi, D. and L. Yariv (2008), "Costly Expertise," American Economic Review, Papers and 
Proceedings, 98(2), 187-193.

Glaeser, E._L.,_B._Sacerdote-and-J.-A.-Scheinkman-(1996), "Grime-aind-Soeial-Interaction;" The Quarterly Journal of Economics, 111(2), 507-548.

Goeree, J., M. A. McConnell, T. Mitchell, T. Tromp, and L. Yariv (2010), "The 1/d Law of Giving," American Economic Journal: Microeconomics, 2(1), 183-203.

Granovetter, M. (1994), Getting a Job: A Study of Contacts and Careers, Northwestern University Press. Evanston.

Greenberg, J., and S. Weber (1986), "Strong Tiebout Equilibrium-under Restricted Preferences Domain," Journal of Economic Theory, 38, 101-117.

Huckfeldt, R. R. and J. Sprague (1995), Citizens, Politics and Social Communication, Cambridge Studies in Public Opinion and Political Psychology.

Kandell, D. B. (1978), "Homophily, Selection and Socialization in Adolescent Friendships," The American Journal of Sociology, 84(2), 427-436.

Katz, E. and P. F. Lazarsfeld (1955), Personal influence: The part played by people in the flow of mass communication, Glencoe, IL: Free Press,

Lazarsfeld, P. F.and R. K. Merton (1954), "Friendship as a social process: a substantive and methodological analysis," in Freedom and Control in Modern Society, ed. M Berger, 18-66. New York: Van Nostrand.

Lipsman A. (2008), "Social Networking Explodes Worldwide as Sites Increase Their Focus on Cultural Relevance," comScore, 12/3/2008.

Lynd, R. S., and H. M. Lynd (1929), Middletown: A Study in Contemporary American Culture. New York, Harcourt, Brace and Company, New York, NY.

Manski, C. F.(1993), "Identification of Endogenous Social Effects: The Reflection Problem," The Review of Economic Studies, 60(3), 531-542.

Manski, C. F. (2000), "Economic Analysis of Social Interactions," The Journal of Economic Perspectives, 14(3), 115-136.

Marmaros, D. and B. Sacerdote (2006), "How do Friendships Form?," The Quarterly Journal of Economics, 121(1), 79-119. 
Mayer, A. and S. L. Puller (2008), "The Old Boy (and Girl) Network: Social Network Formation_on_University_Campuses,"_Journal-of_-_ublic_Eeonomics,-92(1-2),-329-347.

McCarty, C., Killworth, P. D., Bernard, H. R., Johnsen, E., and Shelley, G. (2000), "Comparing Two Methods for Estimating Network Size," Human Organization, 60, 28-39.

McPherson, M., L. Smith-Lovin, and J. Cook (2001), "Birds of a Feather: Homophily in Social Networks," Annual Review of Sociology, 27, 415-444.

Myers, D. G. (2007), Social Psychology, McGraw-Hill, New York, NY.

Peski, M. (2008), "Complementarities, Group Formation and Preferences for Similarity," mimeo.

Rosenblat, T. S. and M. M. Mobius (2004); "Getting Closer or Drifting Apart," Quarterly Journal of Economics, 119(3), 971-1009.

Sproull, L. and S. Kiesler (1991), Connections - New Ways of Working in the Networked Organization, MIT Press, Cambridge, MA.

Thomas, T. L. (2009), "Countering Internet Terrorism," IO Sphere.

Tiebout, C. M. (1956), "A Theory of Local Expenditures," The Journal of Political Economy, $64(5), 416-424$.

Topa, G. (2001), "Social Interactions, Local Spillovers and Unemployment," The Review of Economic Studies, 68(2); 261-295.

Wooders, M., E. Cartwright, and R. Selten (2006), "Behavioral Conformity in Games with Many Players," Games and Economic Behavior, 57, 347-360. 
Bücher des Schwerpunkts Märkte und Politik

Books of the Research Area Markets and Politics

Kai A. Konrad, Holger Zschäpitz

Schulden ohne Sühne: Warum der Absturz der Staatsfinanzen uns alle trifft 2010, C. H. Beck

Áron Kiss

Essays in Political Economy and International Public Finance

2009, Peter Lang Verlag

Kai A. Konrad, Tim Lohse (Eds.)

Einnahmen- und Steuerpolitik in Europa:

Herausforderungen und Chancen

2009, Peter Lang Verlag

Kai A. Konrad

Strategy and Dynamics in Contests

2009, Oxford University Press

Roger D. Congleton, Arye L. Hillman, Kai A. Konrad (Eds.)

40 Years of Research on Rent Seeking

2008, Springer

Kai A. Konrad, Beate Jochimsen (Eds.)

Föderalismuskommission II:

Neuordnung von Autonomie und Verantwortung 2008, Peter Lang Verlag

Mark Gradstein, Kai A. Konrad (Eds.)

Institutions and Norms in Economic Development 2007, MIT Press

Johannes Münster

Mobbers, Robbers, and Warriors

2007, Shaker Verlag

Kai A. Konrad, Beate Jochimsen (Eds.)

Der Föderalstaat nach dem Berlin-Urteil

2007, Peter Lang Verlag

Kai A. Konrad, Beate Jochimsen (Eds.)

Finanzkrise im Bundesstaat

2006, Peter Lang Verlag

Robert Nuscheler

On Competition and Regulation in Health Care

Systems

2005, Peter Lang Verlag

Pablo Beramendi

Decentralization and Income Inequality

2003, Madrid: Juan March Institute

Thomas R. Cusack

A National Challenge at the Local Level: Citizens, Elites and Institutions in Reunified Germany 2003, Ashgate

Sebastian Kessing

Essays on Employment Protection

2003, Freie Universität Berlin

http://www.diss.fu-berlin.de/2003/202
Daniel Krähmer

On Learning and Information in Markets and

Organizations

2003, Shaker Verlag

Tomaso Duso

The Political Economy of the Regulatory Process:

An Empirical Approach

Humboldt-University Dissertation, 2002, Berlin, http://edoc.hu-berlin.de/dissertationen/duso-tomaso2002-07-17/PDF/Duso.pdf

Bob Hancké

Large Firms and Institutional Change. Industrial Renewal and Economic Restructuring in France 2002, Oxford University Press

Andreas Stephan

Essays on the Contribution of Public Infrastructure to Private: Production and its Political Economy

2002, dissertation.de

Peter A. Hall, David Soskice (Eds.)

Varieties of Capitalism

2001, Oxford University Press

Hans Mewis

Essays on Herd Behavior and Strategic Delegation 2001, Shaker Verlag

Andreas Moerke

Organisationslernen über Netzwerke - Die

personellen Verflechtungen von Führungsgremien

japanischer Aktiengesellschaften

2001, Deutscher Universitäts-Verlag

Silke Neubauer

Multimarket Contact and Organizational Design

2001, Deutscher Universitäts-Verlag

Lars-Hendrik Röller, Christian Wey (Eds.)

Die Soziale Marktwirtschaft in der neuen

Weltwirtschaft, WZB Jahrbuch 2001

2001, edition sigma

Michael Tröge

Competition in Credit Markets: A Theoretic

Analysis

2001, Deutscher Universitäts-Verlag

Torben Iversen, Jonas Pontusson, David Soskice

(Eds.)

Unions, Employers, and Central Banks

2000, Cambridge University Press

Tobias Miarka

Financial Intermediation and Deregulation:

A Critical Analysis of Japanese Bank-Firm-

Relationships

2000, Physica-Verlag 
Áron Kiss Coalition Politics and Accountability

Benny Geys Friedrich Heinemann

Alexander Kalb

Salmai Qari

Kai A. Konrad

Benny Geys

Kai A. Konrad Salmai Qari

Sven Chojnacki

Nils Metternich Johannes Münster

Oliver Gürtler Johannes Münster

Dan Kovenock

Brian Roberson

Subhasish M.

Chowdhury

Dan Kovenock

Roman M. Sheremeta

Michael R. Baye

Dan Kovenock Casper G. de Vries

Florian Morath Johannes Münster

Benny Geys

Paolo Buccirossi

Lorenzo Ciari

Tomaso Duso

Giancarlo Spagnolo

Cristiana Vitale

Pedro P. Barros Joseph Clougherty

Jo Seldeslachts

Paolo Buccirossi

Lorenzo Ciari

Tomaso Duso

Giancarlo Spagnolo

Cristiana Vitale

Paolo Buccirossi

Lorenzo Ciari

Tomaso Duso

Giancarlo Spagnolo

Cristiana Vitale

Municipalities Tax Compliance

Mercenaries in Civil Wars, 1950-2000

Sabotage in Dynamic Tournaments

Outcomes

Games

Contests with Rank-Order Spillovers

Information Acquisition in Conflicts

Wars, Presidents and Popularity:

An empirical assessment Policy: Frequency or Composition?

Deterrence in Competition Law
Voter Involvement, Fiscal Autonomy and Public

Sector Efficiency: Evidence from German

Patriotism, Taxation and International Mobility

The Last Refuge of a Scoundrel? Patriotism and

Non-Partisan 'Get-Out-the-Vote' Efforts and Policy

An Experimental Investigation of Colonel Blotto

The Political Cost(s) of War Re-examined

Competition policy and productivity growth:

How to Measure the Deterrence Effects of Merger

Measuring the deterrence properties of competition policy: the Competition Policy Indexes
SP || $2009-05$

SP || $2009-06$

SP II $2009-09$

SP || $2009-10$

SP I| $2009-14$

SP I| $2009-01$

SP || 2009-02

SP II $2009-03$

SP II $2009-04$

SP || $2009-07$

SP || $2009-08$

SP || $2009-11$

SP II $2009-12$

SP || $2009-13$

SP II $2009-15$ 
Talat Mahmood The Decision to Migrate: A Simultaneous Decision

SP || $2009-17$ Klaus Schömann Making Approach 
Dorothea Kübler

Dietmar Fehr

Dorothea Kübler

David Danz

Alastair R. Beresford

Dorothea Kübler

Sören Preibusch

Julia Schmid

Dietmar Fehr

Benny Geys

Margarita Kalamova

Kai A. Konrad

Kai A. Konrad

Florian Morath

Wieland Müller

Kai A. Konrad

Benny Geys

Kai A. Konrad

Kai A. Konrad

Kai A. Konrad

Rabah Amir

Natalia Lazzati

Joseph A Clougherty

Tomaso Duso

Kristof De Witte

Benny Geys

Kai A. Konrad

Florian Morath

Tomaso Duso

Klaus Gugler

Florian Szücs

Florian Morath

Changxia Ke

Kai A. Konrad

Florian Morath

Lydia Mechtenberg Johannes Münster
Experimental Practices in Economics:

SP || $2010-01$

Performativity and the Creation of Phenomena

Information and Beliefs in a Repeated

Normal-form Game

SP II $2010-02$

Unwillingness to Pay for Privacy:

A Field Experiment

SP || $2010-03$

Exclusion in the All-Pay Auction: An Experimental Investigation

War Casualties and US Presidential Popularity: A Comparison of the Korean, Vietnam and Iraq War

Nation Brands and Foreign Direct Investment

Taxation and Market Power

SP || $2010-04$

SP II $2010-05$

SP || $2010-06$

SP || $2010-07$

SP || $2010-08$

Portfolios and Loyal Customers

Federalism and Optimal Allocation Across Levels of Governance

Dynamic Contests

SP || $2010-10$

SP || $2010-11$ Competition

Network Effects, Market Structure and Industry Performance

Using Rival Effects to Identify Synergies and Improve Merger Typologies

Evaluating Efficient Public Good Provision:

Theory and Evidence from a Generalised Conditional Efficiency Model for Public Libraries

Social Mobility and Redistributive Taxation

SP || $2010-15$

An Empirical Assessment of the 2004 EU Merger Policy Reform

SP || $2010-16$

Volunteering and the Strategic Value of Ignorance

SP || $2010-17$

Brothers in Arms - An Experiment on the Alliance Puzzle

SP || $2010-18$

SP || $2010-19$
A strategic mediator who is biased into the same direction as the expert can improve information transmission 
Mariagiovanna

Similarity and Polarization in Groups

SP || $2010-20$

Baccara

Leeat Yariv 
Bei Ihren Bestellungen von WZB-Papers schicken

Sie bitte unbedingt einen an Sie adressierten Auf-

kleber mit sowie je paper eine Briefmarke im Wert

von 0,51 Euro oder einen "Coupon Reponse Inter-

national " (für Besteller aus dem Ausland)
Please send a self addressed label and postage stamps in the amount of 0.51 Euro or a "CouponReponse International" (if you are ordering from outside Germany) for each WZB-paper requested

Absender I Return Address:

Wissenschaftszentrum Berlin

für Sozialforschung

Presse- und Informationsreferat

Reichpietschufer 50

D-10785 Berlin-Tiergarten

Hiermit bestelle ich folgende(s) Discussion paper(s):

Please send me the following Discussion paper(s):

Bestell-Nr. I Order no.

Autor/in, Kurztitel /Author(s) / Title(s) in brief 